\title{
A-QUASICONVEXITY: RELAXATION AND HOMOGENIZATION
}

\author{
Andrea Braides ${ }^{1}$, Irene Fonseca ${ }^{2}$ And Giovanni Leoni ${ }^{3}$
}

Abstract. Integral representation of relaxed energies and of $\Gamma$-limits of functionals

$$
(u, v) \mapsto \int_{\Omega} f(x, u(x), v(x)) d x
$$

are obtained when sequences of fields $v$ may develop oscillations and are constrained to satisfy a system of first order linear partial differential equations. This framework includes the treatement of divergence-free fields, Maxwell's equations in micromagnetics, and curl-free fields. In the latter case classical relaxation theorems in $W^{1, p}$ are recovered.

AMS Subject Classification. 35D99, 35E99, 49J45.

Received March 16, 2000. Revised September 26, 2000.

\section{INTRODUCTION}

In a recent paper Fonseca and Müller [22] have proved that $\mathcal{A}$-quasiconvexity is a necessary and sufficient condition for (sequential) lower semicontinuity of a functional

$$
(u, v) \mapsto \int_{\Omega} f(x, u(x), v(x)) d x,
$$

whenever $f: \Omega \times \mathbb{R}^{m} \times \mathbb{R}^{d} \rightarrow[0, \infty)$ is a Carathéodory integrand satisfying

$$
0 \leq f(x, u, v) \leq a(x, u)\left(1+|v|^{q}\right)
$$

for a.e. $x \in \Omega$ and all $(u, v) \in \mathbb{R}^{m} \times \mathbb{R}^{d}$, where $1 \leq q<\infty, a \in L_{\text {loc }}^{\infty}(\Omega \times \mathbb{R} ;[0, \infty)), \Omega \subset \mathbb{R}^{N}$ is open, bounded, $u_{n} \rightarrow u$ in measure, $v_{n} \rightarrow v$ in $L^{q}\left(\Omega ; \mathbb{R}^{d}\right)$ and $\mathcal{A} v_{n} \rightarrow 0$ in $W^{-1, q}\left(\Omega ; \mathbb{R}^{l}\right)$ (see also [14]). Here, and in what follows, following [32]

$$
\mathcal{A}: L^{q}\left(\Omega ; \mathbb{R}^{d}\right) \rightarrow W^{-1, q}\left(\Omega ; \mathbb{R}^{l}\right), \quad \mathcal{A} v:=\sum_{i=1}^{N} A^{(i)} \frac{\partial v}{\partial x_{i}}
$$

Keywords and phrases: $\mathcal{A}$-quasiconvexity, equi-integrability, Young measure, relaxation, $\Gamma$-convergence, homogenization.

1 SISSA, Trieste, Italy; e-mail: braides@sissa.it

2 Department of MathematicalSciences, Carnegie-Mellon University, Pittsburgh, PA, U.S.A.; e-mail: fonseca@andrew.cmu.edu

3 Dipartimento di Scienze e Tecnologie Avanzate, Università del Piemonte Orientale, Alessandria, Italy;

e-mail: leoni@al.unipmn.it 
is a constant-rank, first order linear partial differential operator, with $A^{(i)}: \mathbb{R}^{d} \rightarrow \mathbb{R}^{l}$ linear transformations, $i=1, \ldots, N$. We recall that $\mathcal{A}$ satisfies the constant-rank property if there exists $r \in \mathbb{N}$ such that

$$
\operatorname{rank} \mathbb{A} w=r \quad \text { for all } w \in S^{N-1},
$$

where

$$
\mathbb{A} w:=\sum_{i=1}^{N} w_{i} A^{(i)}, \quad w \in \mathbb{R}^{N} .
$$

A function $f: \mathbb{R}^{d} \rightarrow \mathbb{R}$ is said to be $\mathcal{A}$-quasiconvex if

$$
f(v) \leq \int_{Q} f(v+w(y)) d y
$$

for all $v \in \mathbb{R}^{d}$ and all $w \in C_{1 \text {-per }}^{\infty}\left(\mathbb{R}^{N} ; \mathbb{R}^{d}\right)$ such that $\mathcal{A} w=0$ and $\int_{Q} w(y) d y=0$. Here $Q$ denotes the unit cube in $\mathbb{R}^{N}$, and the space $C_{1-\text { per }}^{\infty}\left(\mathbb{R}^{N} ; \mathbb{R}^{d}\right)$ is introduced in Section 2 .

The relevance of this general framework, as emphasized by Tartar (see [32,34-39]), lies on the fact that in continuum mechanics and electromagnetism PDEs other than $\operatorname{curl} v=0$ arise naturally, and this calls for a relaxation theory which encompasses PDE constraints of the type $\mathcal{A} v=0$. Some important examples included in this general setting are given by:

(a) [Unconstrained Fields]

$$
\mathcal{A} v \equiv 0 .
$$

Here, due to Jensen's inequality $A$-quasiconvexity reduces to convexity.

(b) [Divergence Free Fields]

where $v: \Omega \subset \mathbb{R}^{N} \rightarrow \mathbb{R}^{N}$ (see [33]).

$$
\mathcal{A} v=0 \quad \text { if and only if } \operatorname{div} v=0,
$$

(c) [Magnetostatics Equations]

$$
\mathcal{A}\left(\begin{array}{c}
m \\
h
\end{array}\right):=\left(\begin{array}{c}
\operatorname{div}(m+h) \\
\operatorname{curl} h
\end{array}\right)=0
$$

where $m: \mathbb{R}^{3} \rightarrow \mathbb{R}^{3}$ is the magnetization and $h: \mathbb{R}^{3} \rightarrow \mathbb{R}^{3}$ is the induced magnetic field (see [17,38]); often these are also called Maxwell's Equations in the micromagnetics literature.

(d) [Gradients]

$$
\mathcal{A} v=0 \quad \text { if and only if } \operatorname{curl} v=0 .
$$

Note that $w \in C_{1-\text { per }}^{\infty}\left(\mathbb{R}^{N} ; \mathbb{R}^{d}\right)$ is such that curl $w=0$ and $\int_{Q} w(y) d y=0$ if and only if there exists $\varphi \in$ $C_{1-p e r}^{\infty}\left(\mathbb{R}^{N} ; \mathbb{R}^{n}\right)$ such that $\nabla \varphi=v$, where $d=n \times N$. Thus in this case we recover the well-known notion of quasiconvexity introduced by Morrey [30].

(e) [Higher Order Gradients]

Replacing the target space $\mathbb{R}^{d}$ by an appropriate finite dimensional vector space $E_{s}^{n}$, it is possible to find a first order linear partial differential operator $\mathcal{A}$ such that $v \in L^{p}\left(\Omega ; E_{s}^{n}\right)$ and $\mathcal{A} v=0$ if and only if there exists $\varphi \in W^{s, q}\left(\Omega ; \mathbb{R}^{n}\right)$ such that $v=\nabla^{s} \varphi$ (see Th. 1.3).

This paper is divided into two parts. In the first part we give an integral representation formula for the relaxed energy in the context of $\mathcal{A}$-quasiconvexity. Precisely, let $1 \leq p<\infty$ and $1<q<\infty$, and consider the functional

defined by

$$
F: L^{p}\left(\Omega ; \mathbb{R}^{m}\right) \times L^{q}\left(\Omega ; \mathbb{R}^{d}\right) \times \mathcal{O}(\Omega) \rightarrow[0, \infty)
$$

$$
F((u, v) ; D):=\int_{D} f(x, u(x), v(x)) d x
$$


where $\mathcal{O}(\Omega)$ is the collection of all open subsets of $\Omega$, and the density $f$ satisfies the following hypothesis:

(H) $f: \Omega \times \mathbb{R}^{m} \times \mathbb{R}^{d} \rightarrow[0, \infty)$ is Carathéodory function satisfying

$$
0 \leq f(x, u, v) \leq C\left(1+|u|^{p}+|v|^{q}\right)
$$

for a.e. $x \in \Omega$ and all $(u, v) \in \mathbb{R}^{m} \times \mathbb{R}^{d}$, and where $C>0$.

For $D \in \mathcal{O}(\Omega)$ and $(u, v) \in L^{p}\left(\Omega ; \mathbb{R}^{m}\right) \times\left(L^{q}\left(\Omega ; \mathbb{R}^{d}\right) \cap \operatorname{ker} \mathcal{A}\right)$ define

$$
\begin{aligned}
\mathcal{F}((u, v) ; D):=\inf \left\{\liminf _{n \rightarrow \infty} F\left(\left(u_{n}, v_{n}\right) ; D\right):\left(u_{n}, v_{n}\right) \in L^{p}\left(D ; \mathbb{R}^{m}\right) \times L^{q}\left(D ; \mathbb{R}^{d}\right),\right. \\
\left.u_{n} \rightarrow u \text { in } L^{p}\left(D ; \mathbb{R}^{m}\right), \quad v_{n} \rightarrow v \text { in } L^{q}\left(D ; \mathbb{R}^{d}\right), \quad \mathcal{A} v_{n} \rightarrow 0 \quad \text { in } W^{-1, q}\left(D ; \mathbb{R}^{l}\right)\right\}
\end{aligned}
$$

It turns out that the condition $\mathcal{A} v_{n} \rightarrow 0$ imposed in (1.2) may be replaced by requiring that $v_{n}$ do satisfy the homogeneous PDE $\mathcal{A} v=0$. Precisely, and in view of Lemma 3.1 and Corollary 3.2 below, it can be shown that

$$
\mathcal{F}((u, v) ; D)=\inf \left\{\liminf _{n \rightarrow \infty} F\left(\left(u, v_{n}\right) ; D\right): v_{n} \in L^{q}\left(D ; \mathbb{R}^{d}\right), v_{n} \rightarrow v \text { in } L^{q}\left(D ; \mathbb{R}^{d}\right), \mathcal{A} v_{n}=0\right\},
$$

and thus

$$
\begin{gathered}
\mathcal{F}((u, v) ; D)=\inf \left\{\liminf _{n \rightarrow \infty} F\left(\left(u_{n}, v_{n}\right) ; D\right):\left(u_{n}, v_{n}\right) \in L^{p}\left(D ; \mathbb{R}^{m}\right) \times L^{q}\left(D ; \mathbb{R}^{d}\right),\right. \\
\left.u_{n} \rightarrow u \text { in } L^{p}\left(D ; \mathbb{R}^{m}\right), \quad v_{n} \rightarrow v \text { in } L^{q}\left(D ; \mathbb{R}^{d}\right), \quad \mathcal{A} v_{n}=0\right\}=: \mathcal{F}_{0}((u, v) ; D) .
\end{gathered}
$$

The first main result of the paper is given by the following theorem:

Theorem 1.1. Under condition $(H)$ and the constant-rank hypothesis (1.1), for all $D \in \mathcal{O}(\Omega), u \in L^{p}\left(\Omega ; \mathbb{R}^{m}\right)$, and $v \in L^{q}\left(\Omega ; \mathbb{R}^{d}\right) \cap \operatorname{ker} \mathcal{A}$, we have

$$
\mathcal{F}((u, v) ; D)=\int_{D} \mathcal{Q}_{\mathcal{A}} f(x, u(x), v(x)) d x
$$

where, for each fixed $(x, u) \in \Omega \times \mathbb{R}^{m}$, the function $\mathcal{Q}_{\mathcal{A}} f(x, u, \cdot)$ is the $\mathcal{A}$-quasiconvexification of $f(x, u, \cdot)$, namely

$$
\mathcal{Q}_{\mathcal{A}} f(x, u, v):=\inf \left\{\int_{Q} f(x, u, v+w(y)) d y: w \in C_{1-\text { per }}^{\infty}\left(\mathbb{R}^{N} ; \mathbb{R}^{d}\right) \cap \operatorname{ker} \mathcal{A}, \int_{Q} w(y) d y=0\right\}
$$

for all $v \in \mathbb{R}^{d}$.

Remarks 1.2. (i) Note that in the degenerate case where $\mathcal{A}=0, \mathcal{A}$-quasiconvex functions are convex and Theorem 1.1 together with condition (1.4) yield a convex relaxation result with respect to $L^{p} \times L^{q}$ (weak) convergence. See the monograph of Buttazzo [12] for related results in this context.

(ii) If the function $f$ also satisfies a growth condition of order $q$ from below in the variable $v$, that is

$$
f(x, u, v) \geq \frac{1}{C}|v|^{q}-C
$$


for a.e. $x \in \Omega$ and all $(u, v) \in \mathbb{R}^{m} \times \mathbb{R}^{d}$, then a simple diagonalization argument shows that $(u, v) \mapsto \mathcal{F}((u, v) ; D)$ is $L^{p} \times\left(L^{q}\right.$-weak) lower semicontinuous, i.e.,

$$
\int_{D} \mathcal{Q}_{\mathcal{A}} f(x, u(x), v(x)) d x \leq \liminf _{n \rightarrow \infty} \int_{D} \mathcal{Q}_{\mathcal{A}} f\left(x, u_{n}(x), v_{n}(x)\right) d x
$$

whenever $u_{n} \in L^{p}\left(\Omega ; \mathbb{R}^{m}\right), v_{n} \in L^{q}\left(\Omega ; \mathbb{R}^{d}\right) \cap \operatorname{ker} \mathcal{A}, u_{n} \rightarrow u$ in $L^{p}\left(D ; \mathbb{R}^{m}\right), v_{n} \rightarrow v$ in $L^{q}\left(D ; \mathbb{R}^{d}\right)$. In particular $\mathcal{Q}_{\mathcal{A}} f$ is $\mathcal{A}$-quasiconvex if $f$ is continuous and

$$
\frac{1}{C}|v|^{q}-C \leq f(v) \leq C\left(1+|v|^{q}\right)
$$

for some $C>0$, and all $v \in \mathbb{R}^{d}$ (see the proof of Cor. 5.7).

The lower semicontinuity result (1.5) is not covered by Theorem 3.7 in [22], where it is assumed that the integrand be $\mathcal{A}$-quasiconvex and continuous in the $v$ variable. However, as remarked in [22], in the realm of general $\mathcal{A}$-quasiconvexity the function $\mathcal{Q}_{\mathcal{A}} f(x, u, \cdot)$ may not be continuous, even if $f(x, u, \cdot)$ is. Indeed in the degenerate case $\operatorname{ker} \mathcal{A}=\{0\}$ all functions are $\mathcal{A}$-quasiconvex. Also, when $N=1, d=2$, and $v=\left(v_{1}, v_{2}\right)$, consider

Then for $w \in \mathbb{R}$

$$
\mathcal{A} v:=\left(\begin{array}{ll}
0 & 1
\end{array}\right)\left(\begin{array}{l}
v_{1}^{\prime} \\
v_{2}^{\prime}
\end{array}\right)
$$

$$
\mathbb{A} w=\left(\begin{array}{ll}
0 & w
\end{array}\right)
$$

and thus when $|w|=1$ the matrix $\mathbb{A} w$ has constant rank 1 . For any given function $f(v)$ the $\mathcal{A}$-quasiconvex envelope of $f$ is obtained by convexification in the first component, so that by considering e.g. $(c f .[22,28])$

$$
f_{1}(v):=e^{-\left|v_{1}\right| v_{2}^{2}}, \quad f_{2}(v):=\left(1+\left|v_{1}\right|\right)^{\left|v_{2}\right|},
$$

one gets

$$
\mathcal{Q}_{\mathcal{A}} f_{1}(v)=\left\{\begin{array}{ll}
0 & \text { if } v_{2} \neq 0 \\
1 & \text { if } v_{2}=0
\end{array}, \quad \mathcal{Q}_{\mathcal{A}} f_{1}(v)= \begin{cases}\left(1+\left|v_{1}\right|\right)^{\left|v_{2}\right|} & \text { if }\left|v_{2}\right| \geq 1 \\
1 & \text { if }\left|v_{2}\right|<1\end{cases}\right.
$$

(iii) The continuity of $f$ with respect to $v$ is essential to ensure the representation of $\mathcal{F}$ provided in Theorem 1.1, in contrast with the case where $\mathcal{A} v=0$ if and only if $\operatorname{curl} v=0$. In fact, if $f: \mathbb{R}^{n \times N} \rightarrow[0, \infty)$ is a Borel function satisfying the growth condition

$$
0 \leq f(v) \leq C\left(1+|v|^{q}\right)
$$

for $C>0,1 \leq q<\infty, v \in \mathbb{R}^{n \times N}$, then it can be shown easily that

$$
\mathcal{F}(w ; D)=\int_{D} \mathcal{Q} f(\nabla w(x)) d x
$$

for all $D \in \mathcal{O}(\Omega), w \in W^{1, q}\left(\Omega ; \mathbb{R}^{n}\right)$, where $\mathcal{Q} f$ is the quasiconvex envelope of $f$. Indeed, $Q f$ is a (continuous) quasiconvex function satisfying $(H)$ (see [18], [8] Th. 4.3); therefore by Theorem 1.1

$$
w \mapsto \int_{D} Q f(\nabla w(x)) d x
$$

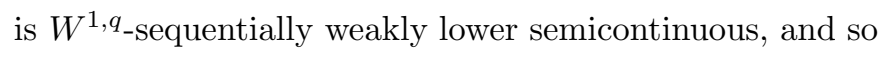

$$
\int_{D} Q f(\nabla w(x)) d x \leq \mathcal{F}(w ; D) .
$$


Conversely, under hypothesis $(H)$ it is known that $\mathcal{F}(v ; \cdot)$ admits an integral representation (see Th. 9.1 in [10], Th. 20.1 in [15])

$$
\mathcal{F}(w ; D)=\int_{D} \varphi(\nabla w(x)) d x
$$

where $\varphi$ is a quasiconvex function, and $\varphi(v) \leq f(v)$ for all $v \in \mathbb{R}^{n \times N}$. Hence $\varphi \leq Q f$ and we conclude that (1.6) holds.

For general constant-rank operators $\mathcal{A}$, and if $f$ is not continuous with respect to $v$, it may happen that $\mathcal{F}_{0}((u, v) ; \cdot)$ is not even the trace of a Radon measure in $\mathcal{O}(\Omega)$ and thus (1.3) fails. As an example, consider $d=2, N=1, \Omega:=(0,1), v=\left(v_{1}, v_{2}\right)$, and let $\mathcal{A}(v)=0$ if and only if $v_{2}^{\prime}=0$ as in (ii) above. Let

$$
f(v):= \begin{cases}\left(v_{1}-1\right)^{2}+v_{2}^{2}, & \text { if } v_{2} \in \mathbb{Q} \\ \left(v_{1}+1\right)^{2}+v_{2}^{2}, & \text { if } v_{2} \notin \mathbb{Q} .\end{cases}
$$

Although $f$ satisfies a quadratic growth condition of the type $(H)$, and $\left(A_{3}\right)$ holds with $q=2$, it is easy to see that for all intervals $(a, b) \subset(0,1)$,

$$
\mathcal{F}_{0}((u, v) ;(a, b))=\mathcal{F}_{0}(v ;(a, b))=\min \left\{\int_{a}^{b}\left(\left(v_{1}-1\right)^{2}+v_{2}^{2}\right) d x, \int_{a}^{b}\left(\left(v_{1}+1\right)^{2}+v_{2}^{2}\right) d x\right\}
$$

which is not the trace of a Radon measure on $\mathcal{O}(\Omega)$. On the other hand, it may be shown that (see the Appendix below for a proof)

$$
\mathcal{F}((u, v) ;(a, b))=\mathcal{F}(v ;(a, b))=\int_{a}^{b}\left(\psi^{* *}\left(v_{1}\right)+v_{2}^{2}\right) d x
$$

where $\psi^{* *}\left(v_{1}\right)$ is the convex envelope of

$$
\psi\left(v_{1}\right):=\min \left\{\left(v_{1}-1\right)^{2},\left(v_{1}+1\right)^{2}\right\} .
$$

(iv) Using the growth condition $(H)$, a mollification argument, and the linearity of $\mathcal{A}$, it can be shown that (see Rem. 3.3 in [22])

$$
\mathcal{Q}_{\mathcal{A}} f(x, u, v)=\inf \left\{\int_{Q} f(x, u, v+w(y)) d y: w \in L_{1-\text { per }}^{q}\left(\mathbb{R}^{N} ; \mathbb{R}^{d}\right) \cap \operatorname{ker} \mathcal{A}, \quad \int_{Q} w(y) d y=0\right\}
$$

We write $w \in L_{1 \text {-per }}^{q}\left(\mathbb{R}^{N} ; \mathbb{R}^{d}\right) \cap \operatorname{ker} \mathcal{A}$ when $w \in L_{1 \text {-per }}^{q}\left(\mathbb{R}^{N} ; \mathbb{R}^{d}\right)$ and $\mathcal{A} w=0$ in $W^{-1, q}\left(Q ; \mathbb{R}^{l}\right)$.

(v) We may also treat the cases $q=1, \infty$ and $p=\infty$. See Theorem 3.6 below.

The proof of Theorem 1.1 relies heavily on the use of Young measures (see [5, 40]). However, instead of applying directly the arguments of Fonseca and Müller [22] (based on Balder's [4] and Kristensen's [26] approach in the curl-free case), we use these together with the blow-up method introduced by Fonseca and Müller in [20].

Although in Theorem 1.1 the functions $u$ and $v$ are not related to each other, the arguments of the proof work equally well when $u$ and $v$ are not independent. Indeed as a corollary, we can prove the following two theorems:

Theorem 1.3. Let $1 \leq p \leq \infty, s \in \mathbb{N}$, and suppose that $f: \Omega \times E_{[s-1]}^{n} \times E_{s}^{n} \rightarrow[0, \infty)$ is a Carathéodory function satisfying

$$
0 \leq f(x, \mathbf{u}, v) \leq C\left(1+|\mathbf{u}|^{p}+|v|^{p}\right), \quad 1 \leq p<\infty
$$

for a.e. $x \in \Omega$ and all $(\mathbf{u}, v) \in E_{[s-1]}^{n} \times E_{s}^{n}$, where $C>0$, and

$$
\chi_{\Omega} f \in L_{\mathrm{loc}}^{\infty}\left(\mathbb{R}^{N} \times E_{[s-1]}^{n} \times E_{s}^{n} ;[0, \infty)\right) \quad \text { if } p=\infty
$$


Then for every $u \in W^{s, p}\left(\Omega ; \mathbb{R}^{n}\right)$ we have

$$
\begin{array}{r}
\int_{\Omega} \mathcal{Q}^{s} f\left(x, u, \ldots, \nabla^{s} u\right) d x=\inf \left\{\liminf _{k \rightarrow \infty} \int_{\Omega} f\left(x, u_{k}, \ldots, \nabla^{s} u_{k}\right) d x:\left\{u_{k}\right\} \subset W^{s, p}\left(\Omega ; \mathbb{R}^{n}\right),\right. \\
\left.u_{k} \rightarrow u \text { in } W^{s, p}\left(\Omega ; \mathbb{R}^{n}\right) \quad(\stackrel{\star}{\rightarrow} \text { if } p=\infty)\right\},
\end{array}
$$

where, for a.e. $x \in \Omega$ and all $(\mathbf{u}, v) \in E_{[s-1]}^{n} \times E_{s}^{n}$,

$$
\mathcal{Q}^{s} f(x, \mathbf{u}, v):=\inf \left\{\int_{Q} f\left(x, \mathbf{u}, v+\nabla^{s} w(y)\right) d y: w \in C_{1-\text { per }}^{\infty}\left(\mathbb{R}^{N} ; \mathbb{R}^{n}\right)\right\}
$$

Remarks 1.4. (i) Here $E_{s}^{n}$ stands for the space of $n$-tuples of symmetric $s$-linear maps on $\mathbb{R}^{\mathbb{N}}$,

$$
E_{[s-1]}^{n}:=\mathbb{R}^{n} \times E_{1}^{n} \times \cdots \times E_{s-1}^{n},
$$

and

$$
\nabla^{l} u:=\left(\frac{\partial^{l} u}{\partial x_{1}^{\alpha_{1}} \ldots \partial x_{N}^{\alpha_{N}}}\right)_{\alpha_{1}+\ldots+\alpha_{N}=l}, \quad l \geq 1
$$

(ii) When $s=1$ we recover classical relaxation results (see e.g. the work of Acerbi and Fusco [1], Dacorogna [13], Marcellini and Sbordone [28] and the references contained therein).

When $s>1$ lower semicontinuity results related to Theorem 1.3 are due to Meyers [29], Fusco [23] and Guidorzi and Poggiolini [25], while we are not aware of any integral representation formula for the relaxed energy, when the integrand depends on the full set of variables, that is $f=f\left(x, u, \ldots, \nabla^{s} u\right)$. This is due to the fact that classical truncation methods for $s=1$ cannot be extended in a simple way to truncate higher order derivatives. The results of Fonseca and Müller (see the proof of Lem. 2.15 in [22]), where the truncation is only on the highest order derivative $\nabla^{s} u$, and Corollary 3.2 below, allows us to overcome this difficulty. Note however that this technique relies heavily on $p$-equi-integrability, and thus cannot work in the case $p=1$, if one replaces weak convergence in $W^{s, 1}\left(\Omega ; \mathbb{R}^{n}\right)$ with the natural convergence, which is strong convergence in $W^{s-1,1}\left(\Omega ; \mathbb{R}^{n}\right)$. In this context, a relaxation result has been given by Amar and De Cicco [2], but only when $f=f\left(\nabla^{s} u\right)$, so that truncation is not needed. The general case where $f$ depends also on lower order derivatives has been addressed by Fonseca et al. [19].

Theorem 1.5. Let $1 \leq p \leq \infty$, let $\Omega \subset \mathbb{R}^{N}$ be an open, bounded, connected set, and suppose that $f: \Omega \times \mathbb{R}^{N} \times$ $\mathbb{R}^{N^{2}} \rightarrow[0, \infty)$ is a Carathéodory function satisfying

$$
0 \leq f(x, u, v) \leq C\left(1+|u|^{p}+|v|^{p}\right), \quad 1 \leq p<\infty
$$

for a.e. $x \in \Omega$ and all $(u, v) \in \mathbb{R}^{N} \times \mathbb{R}^{N^{2}}$, where $C>0$, and

$$
\chi_{\Omega} f \in L_{\mathrm{loc}}^{\infty}\left(\mathbb{R}^{N} \times \mathbb{R}^{N} \times \mathbb{R}^{N^{2}} ;[0, \infty)\right) \quad \text { if } p=\infty .
$$

Then for every $u \in W^{1, p}\left(\Omega ; \mathbb{R}^{N}\right)$ such that $\operatorname{div} u=0$, we have

$$
\begin{gathered}
\int_{\Omega} \bar{f}(x, u(x), \nabla u(x)) d x=\inf \left\{\liminf _{n \rightarrow \infty} \int_{\Omega} f\left(x, u_{n}(x), \nabla u_{n}(x)\right) d x:\left\{u_{n}\right\} \subset W^{1, p}\left(\Omega ; \mathbb{R}^{N}\right),\right. \\
\left.\operatorname{div} u_{n}=0, \quad u_{n} \rightarrow u \text { in } W^{1, p}\left(\Omega ; \mathbb{R}^{N}\right) \quad(\stackrel{\star}{\rightarrow} \text { if } p=\infty)\right\},
\end{gathered}
$$


where, for a.e. $x \in \Omega$ and all $(u, v) \in \mathbb{R}^{N} \times \mathbb{R}^{N^{2}}$,

$$
\bar{f}(x, u, v):=\inf \left\{\int_{Q} f(x, u, v+\nabla w(y)) d y: w \in C_{1-\text { per }}^{\infty}\left(\mathbb{R}^{N} ; \mathbb{R}^{N}\right), \operatorname{div} w=0\right\}
$$

Remark 1.6. To the authors' knowledge, this result is new in this generality (for a different proof, with additional smoothness assumptions, see [9]). A related problem was addressed by Dal Maso et al. in [16], where it was shown that the $\Gamma$-limit of a family of functionals of the type (1.7) may be non local if $(H)$ is violated.

In the second part of the paper we present ( $\Gamma$-convergence) homogenization results for periodic integrands in the context of $\mathcal{A}$-quasiconvexity. Let $\varepsilon>0$ and $1<q<\infty$, and consider a family of functionals

$$
\mathcal{F}_{\varepsilon}:\left(L^{q}\left(\Omega ; \mathbb{R}^{d}\right) \cap \operatorname{ker} \mathcal{A}\right) \times \mathcal{O}(\Omega) \rightarrow[0, \infty)
$$

defined by

$$
\mathcal{F}_{\varepsilon}(v ; D):=\int_{D} f\left(\frac{x}{\varepsilon}, v(x)\right) d x
$$

where the density $f$ satisfies the following hypotheses:

$\left(A_{1}\right) f: \mathbb{R}^{N} \times \mathbb{R}^{d} \rightarrow[0, \infty)$ is a continuous function, $Q$-periodic in the first argument, that is $f\left(x+e_{i}, v\right)=f(x, v)$ for every $i=1, \ldots, N$, where $e_{i}$ are the elements of the canonical basis of $\mathbb{R}^{N}$;

$\left(A_{2}\right)$ there exists $C>0$ such that

$$
0 \leq f(x, v) \leq C\left(1+|v|^{q}\right)
$$

for all $(x, v) \in \mathbb{R}^{N} \times \mathbb{R}^{d}$;

$\left(A_{3}\right)$ there exists $C>0$ such that

for all $(x, v) \in \mathbb{R}^{N} \times \mathbb{R}^{d}$.

$$
f(x, v) \geq \frac{1}{C}|v|^{q}-C
$$

Let $\varepsilon_{n} \rightarrow 0^{+}$. We say that a functional

$$
\mathcal{J}:\left(L^{q}\left(\Omega ; \mathbb{R}^{d}\right) \cap \operatorname{ker} \mathcal{A}\right) \times \mathcal{O}(\Omega) \rightarrow[0,+\infty]
$$

is the $\Gamma-\lim \inf \left(\right.$ resp. $\Gamma-\limsup$ ) of the sequence of functionals $\left\{\mathcal{F}_{\varepsilon_{n}}\right\}$ with respect to the weak convergence in $L^{q}\left(\Omega ; \mathbb{R}^{d}\right)$ if for every $v \in L^{q}\left(\Omega ; \mathbb{R}^{d}\right) \cap \operatorname{ker} \mathcal{A}$

$$
\begin{array}{r}
\mathcal{J}(v ; \Omega)=\inf \left\{\liminf _{n \rightarrow \infty}\left(\text { resp. } \limsup _{n \rightarrow \infty}\right) \mathcal{F}_{\varepsilon_{n}}\left(v_{n} ; \Omega\right): v_{n} \in L^{q}\left(\Omega ; \mathbb{R}^{d}\right) \cap \operatorname{ker} \mathcal{A}\right. \\
\left.v_{n} \rightarrow v \operatorname{in} L^{q}\left(\Omega ; \mathbb{R}^{d}\right)\right\}
\end{array}
$$

and we write

$$
\mathcal{J}=\Gamma-\liminf _{n \rightarrow \infty} \mathcal{F}_{\varepsilon_{n}}\left(\text { resp. } \mathcal{J}=\Gamma-\limsup _{n \rightarrow \infty} \mathcal{F}_{\varepsilon_{n}}\right) .
$$

When finite energy sequences are $L^{q}$-equibounded then the infimum in the definition of $\Gamma-\lim$ inf (resp. $\Gamma-\limsup )$ is attained. We say that the sequence $\left\{\mathcal{F}_{\varepsilon_{n}}\right\} \Gamma$-converges to $\mathcal{J}$ if the $\Gamma-\lim \inf$ and $\Gamma-\lim$ sup coincide, and we write

$$
\mathcal{J}=\Gamma-\lim _{n \rightarrow \infty} \mathcal{F}_{\varepsilon_{n}} .
$$


The functional $\mathcal{J}$ is said to be the $\Gamma-\liminf \left(\right.$ resp. $\Gamma-\limsup$ ) of the family of functionals $\left\{\mathcal{F}_{\varepsilon}\right\}$ with respect to the weak convergence in $L^{q}\left(\Omega ; \mathbb{R}^{d}\right)$ if for every sequence $\varepsilon_{n} \rightarrow 0^{+}$we have that

$$
\mathcal{J}=\Gamma-\liminf _{n \rightarrow \infty} \mathcal{F}_{\varepsilon_{n}} \quad\left(\text { resp. } \mathcal{J}=\Gamma-\limsup _{n \rightarrow \infty} \mathcal{F}_{\varepsilon_{n}}\right)
$$

and we write

$$
\mathcal{J}=\Gamma-\liminf _{\varepsilon \rightarrow 0} \mathcal{F}_{\varepsilon} \quad\left(\text { resp. } \mathcal{J}=\Gamma-\limsup _{\varepsilon \rightarrow 0} \mathcal{F}_{\varepsilon}\right) .
$$

Finally, we say that $\mathcal{J}$ is the is the $\Gamma$-limit of the family of functionals $\left\{\mathcal{F}_{\varepsilon}\right\}$, and we write

$$
\mathcal{J}=\Gamma-\lim _{n \rightarrow \infty} \mathcal{F}_{\varepsilon_{n}}
$$

if $\Gamma-\lim \inf$ and $\Gamma-\lim \sup$ coincide.

In the sequel we will also consider functionals $\mathcal{J}$ given by (1.8) where we replace the weak convergence $v_{n} \rightarrow v$ with the convergence $v_{n} \rightarrow v$ with respect to some metric $d$. In order to highlight this dependence on the metric $d$ these functionals will be denoted as

$$
\mathcal{J}=\Gamma(d)-\liminf _{n \rightarrow \infty} \mathcal{F}_{\varepsilon_{n}} \quad\left(\text { resp. } \mathcal{J}=\Gamma(d)-\limsup _{n \rightarrow \infty} \mathcal{F}_{\varepsilon_{n}}\right),
$$

as it is customary (see $[10,15])$.

Theorem 1.7. Under hypotheses $\left(A_{1}\right)-\left(A_{2}\right)$ and the constant-rank hypothesis (1.1),

$$
\mathcal{F}_{\text {hom }}=\Gamma-\liminf _{\varepsilon \rightarrow 0} \mathcal{F}_{\varepsilon}
$$

where

$$
\mathcal{F}_{\text {hom }}(v ; D):=\int_{D} f_{\text {hom }}(v) d x
$$

for all $v \in L^{q}\left(\Omega ; \mathbb{R}^{d}\right) \cap \operatorname{ker} \mathcal{A}$ and $D \in \mathcal{O}(\Omega)$, and

$$
f_{\text {hom }}(v):=\inf _{k \in \mathbb{N}} \frac{1}{k^{N}} \inf \left\{\int_{k Q} f(x, v+w(x)) d x: \quad w \in L_{k \text {-per }}^{q}\left(\mathbb{R}^{N} ; \mathbb{R}^{d}\right) \cap \operatorname{ker} \mathcal{A}, \quad \int_{k Q} w(x) d x=0\right\}
$$

for all $v \in \mathbb{R}^{d}$. Moreover, if $\left(A_{3}\right)$ holds then

$$
\mathcal{F}_{\text {hom }}=\Gamma-\lim _{\varepsilon \rightarrow 0} \mathcal{F}_{\varepsilon} .
$$

For the definition of the space $L_{k \text {-per }}^{q}\left(\mathbb{R}^{N} ; \mathbb{R}^{d}\right)$, we direct the reader to Section 2 .

Remarks 1.8. (i) Using the growth condition $\left(A_{2}\right)$, a mollification argument, and the linearity of $\mathcal{A}$, it can be shown that

$$
\begin{array}{r}
f_{\text {hom }}(v)=\inf _{k \in \mathbb{N}} \frac{1}{k^{N}} \inf \left\{\int_{k Q} f(x, v+w(x)) d x: \quad w \in L_{k \text {-per }}^{\infty}\left(\mathbb{R}^{N} ; \mathbb{R}^{d}\right) \cap \operatorname{ker} \mathcal{A},\right. \\
\left.\int_{k Q} w(x) d x=0\right\} .
\end{array}
$$

See also Corollary 5.7 below.

(ii) When $f$ satisfies the $q$-Lipschitz condition

$$
\left|f\left(x, v_{1}\right)-f\left(x, v_{2}\right)\right| \leq C\left(\left|v_{1}\right|^{q-1}+\left|v_{2}\right|^{q-1}+1\right)\left|v_{1}-v_{2}\right|
$$


for all $x \in \mathbb{R}^{N}, v_{1}, v_{2} \in \mathbb{R}^{d}$, and for some $C>0$, then the continuity of $f(\cdot, v)$ can be weakened to measurability, namely $f$ can be assumed to be simply Carathéodory. Note that (1.10) is not restrictive when $\mathcal{A}=$ curl, that is when $v=\nabla u$ for some $u \in W^{1, q}\left(\Omega ; \mathbb{R}^{m}\right), d=N \times m$. Indeed, in this case in the definition of $\Gamma$-convergence we may replace the weak convergence of the gradients in $L^{q}\left(\Omega ; \mathbb{R}^{d}\right)$ with the strong convergence in $L^{q}\left(\Omega ; \mathbb{R}^{m}\right)$ of the potentials normalized to have zero average over $\Omega$, and thus

$$
\begin{aligned}
\Gamma-\lim _{\varepsilon \rightarrow 0} \int_{\Omega} f\left(\frac{x}{\varepsilon}, v(x)\right) d x & =\Gamma\left(L^{q}\left(\Omega ; \mathbb{R}^{m}\right)\right)-\lim _{\varepsilon \rightarrow 0} \int_{\Omega} f\left(\frac{x}{\varepsilon}, \nabla u(x)\right) d x \\
& =\Gamma\left(L^{q}\left(\Omega ; \mathbb{R}^{m}\right)\right)-\lim _{\varepsilon \rightarrow 0} \int_{\Omega} \mathcal{Q} f\left(\frac{x}{\varepsilon}, \nabla u(x)\right) d x
\end{aligned}
$$

by Proposition 7.13 in [10]. As shown in [27], if $f(x, v)$ is a Borel function which satisfies the growth condition $\left(A_{2}\right)$ then its quasiconvex envelope $\mathcal{Q} f$ satisfies $(1.10)$.

A similar argument fails for general $\mathcal{A}$-quasiconvexity, since the function $\mathcal{Q}_{\mathcal{A}} f(x, \cdot)$ may not even be continuous, see Remark 1.2(i) above.

In Section 2 we collect preliminary results on Young measures and $\Gamma$-convergence. The general relaxation results (see Th. 1.1 and its exstension Th. 3.6) are proved in Section 3, and Section 4 is devoted to the applications of the general relaxation principle to Theorems 1.3 and 1.5. Finally, in Section 5 we address homogenization of functionals of $\mathcal{A}$-constrained vector fields.

\section{Preliminaries}

We start with some notation. Here $\Omega$ is an open, bounded subset of $\mathbb{R}^{N}, \mathcal{L}^{N}$ is the $N$ dimensional Lebesgue measure, $S^{N-1}:=\left\{x \in \mathbb{R}^{N}:|x|=1\right\}$ is the unit sphere, and $Q:=(-1 / 2,1 / 2)^{N}$ the unit cube centered at the origin. We set $Q\left(x_{0}, \varepsilon\right):=x_{0}+\varepsilon Q$ for $\varepsilon>0$ and $x_{0} \in \mathbb{R}^{N}$. A function $w \in L_{\text {loc }}^{q}\left(\mathbb{R}^{N} ; \mathbb{R}^{d}\right)$ is said to be $Q-$ periodic if $w\left(x+e_{i}\right)=w(x)$ for a.e. all $x \in \mathbb{R}^{N}$ and every $i=1, \ldots, N$, where $\left(e_{1}, \ldots, e_{N}\right)$ is the canonical basis of $\mathbb{R}^{N}$. We write $w \in L_{1-\text { per }}^{q}\left(\mathbb{R}^{N} ; \mathbb{R}^{d}\right)$. More generally, $w \in L_{\text {loc }}^{q}\left(\mathbb{R}^{N} ; \mathbb{R}^{d}\right)$ is said to be $k Q$-periodic, $k \in \mathbb{N}$, if $w(k \cdot)$ is $Q$-periodic. We write $w \in L_{k \text {-per }}^{q}\left(\mathbb{R}^{N} ; \mathbb{R}^{d}\right)$. Also $C_{1 \text {-per }}^{\infty}\left(\mathbb{R}^{N} ; \mathbb{R}^{d}\right)$ will stands for the space of $Q-$ periodic functions in $C^{\infty}\left(\mathbb{R}^{N} ; \mathbb{R}^{d}\right)$.

We recall briefly some facts about Young measures which will be useful in the sequel (see e.g. [5, 33]). If $D$ is an open set (not necessarily bounded), we denote by $C_{c}\left(D ; \mathbb{R}^{d}\right)$ the set of continuous functions with compact support in $D$, endowed with the supremum norm. The dual of the closure of $C_{c}\left(D ; \mathbb{R}^{d}\right)$ may be identified with the set of $\mathbb{R}^{d}$-valued Radon measures with finite mass $\mathcal{M}\left(D ; \mathbb{R}^{d}\right)$, through the duality

$$
\langle\nu, f\rangle:=\int_{D} f(y) d \nu(y), \quad \nu \in \mathcal{M}\left(D ; \mathbb{R}^{d}\right), \quad f \in C_{c}\left(D ; \mathbb{R}^{d}\right)
$$

A map $\nu: \Omega \rightarrow \mathcal{M}\left(D ; \mathbb{R}^{d}\right)$ is said to be weak-* measurable if $x \mapsto\left\langle\nu_{x}, f\right\rangle$ are measurable for all $f \in C_{c}\left(D ; \mathbb{R}^{d}\right)$.

The following result is a corollary of the Fundamental Theorem on Young Measures (see $[5,7,34]$ )

Theorem 2.1. Let $z_{n}: \Omega \rightarrow \mathbb{R}^{d}$ be measurable functions such that

$$
\sup _{n \in \mathbb{N}} \int_{\Omega}\left|z_{n}\right|^{q} d x<\infty
$$

for some $q>0$. Then there exists a subsequence $\left\{z_{n_{k}}\right\}$ of $\left\{z_{n}\right\}$ and a weak-* measurable map $\nu: \Omega \rightarrow \mathcal{M}\left(\mathbb{R}^{d} ; \mathbb{R}^{d}\right)$ such that

(i) $\nu_{x} \geq 0,\left\|\nu_{x}\right\|_{\mathcal{M}}=\int_{\mathbb{R}^{d}} d \nu_{x}=1$ for a.e. $x \in \Omega$; 
(ii) if $f: \Omega \times \mathbb{R}^{d} \rightarrow \mathbb{R}$ is a normal function bounded from below then

$$
\liminf _{k \rightarrow \infty} \int_{\Omega} f\left(x, z_{n_{k}}(x)\right) d x \geq \int_{\Omega} \bar{f}(x) d x<\infty
$$

where

$$
\bar{f}(x):=\left\langle\nu_{x}, f(x, \cdot)\right\rangle=\int_{\mathbb{R}^{d}} f(x, y) d \nu_{x}(y) ;
$$

(iii) for any Carathéodory function $f: \Omega \times \mathbb{R}^{d} \rightarrow \mathbb{R}$ bounded from below one has

$$
\lim _{k \rightarrow \infty} \int_{\Omega} f\left(x, z_{n_{k}}(x) d x=\int_{\Omega} \bar{f}(x) d x<\infty\right.
$$

if and only if $\left\{f\left(\cdot, z_{n_{k}}(\cdot)\right)\right\}$ is equi-integrable.

The map $\nu: \Omega \rightarrow \mathcal{M}\left(\mathbb{R}^{d} ; \mathbb{R}^{d}\right)$ is called the Young measure generated by the sequence $\left\{z_{n_{k}}\right\}$.

Proposition 2.2. If $\left\{z_{n}\right\}$ generates a Young measure $\nu$ and $v_{n} \rightarrow 0$ in measure, then $\left\{z_{n}+v_{n}\right\}$ still generates the Young measure $\nu$.

If $1<q \leq \infty$ then $W^{-1, q}\left(\Omega ; \mathbb{R}^{l}\right)$ is the dual of $W_{0}^{1, q^{\prime}}\left(\Omega ; \mathbb{R}^{l}\right)$, where $q^{\prime}$ is the Hölder conjugate exponent of $q$, that is $1 / q+1 / q^{\prime}=1$. It is well known that $F \in W^{-1, q}\left(\Omega ; \mathbb{R}^{l}\right)$ if and only if there exist $g_{1}, \ldots, g_{N} \in L^{q}\left(\Omega ; \mathbb{R}^{l}\right)$ such that

$$
\langle F, w\rangle=\sum_{i=1}^{N} \int_{\Omega} g_{i} \cdot \frac{\partial w}{\partial x_{i}} d x \quad \text { for all } w \in W_{0}^{1, q}\left(\Omega ; \mathbb{R}^{l}\right) .
$$

Consider a collection of linear operators $A^{(i)}: \mathbb{R}^{d} \rightarrow \mathbb{R}^{l}, i=1, \ldots, N$, and define the differential operator

$$
\begin{aligned}
\mathcal{A}: L^{q}\left(\Omega ; \mathbb{R}^{d}\right) & \longrightarrow W^{-1, q}\left(\Omega ; \mathbb{R}^{l}\right) \\
v & \longmapsto \mathcal{A} v
\end{aligned}
$$

as follows:

$$
\langle\mathcal{A} v, w\rangle:=\left\langle\sum_{i=1}^{N} A^{(i)} \frac{\partial v}{\partial x_{i}}, w\right\rangle=-\sum_{i=1}^{N} \int_{\Omega} A^{(i)} v \frac{\partial w}{\partial x_{i}} d x \quad \text { for all } w \in W_{0}^{1, q}\left(\Omega ; \mathbb{R}^{l}\right) .
$$

Even though the operator $\mathcal{A}$ so defined depends on $\Omega$, we will omit reference to the underlying domain whenever it is clear from the context. In particular, if $v \in L_{1 \text {-per }}^{q}\left(\mathbb{R}^{N} ; \mathbb{R}^{d}\right)$ then we will say that $v \in \operatorname{ker} \mathcal{A}$ if $\mathcal{A} v=0$ in $W^{-1, q}\left(Q ; \mathbb{R}^{l}\right)$.

Throughout the paper we assume that $\mathcal{A}$ satisfies the constant-rank property (1.1).

The following proposition is due to Fonseca and Müller [22].

Proposition 2.3. (i) $(1<q<+\infty)$ Let $1<q<+\infty$, let $\left\{V_{n}\right\}$ be a bounded sequence in $L^{q}\left(\Omega ; \mathbb{R}^{d}\right)$ such that $\mathcal{A} V_{n} \rightarrow 0$ in $W^{-1, q}\left(\Omega ; \mathbb{R}^{l}\right), V_{n} \rightarrow V$ in $L^{q}\left(\Omega ; \mathbb{R}^{d}\right)$, and assume that $\left\{V_{n}\right\}$ generates a Young measure $\nu$. Then there exists a q-equi-integrable sequence $\left\{v_{n}\right\} \subset L^{q}\left(\Omega ; \mathbb{R}^{d}\right) \cap \operatorname{ker} \mathcal{A}$ such that

$$
\int_{\Omega} v_{n} d x=\int_{\Omega} V d x, \quad\left\|v_{n}-V_{n}\right\|_{L^{s}(\Omega)} \rightarrow 0 \quad \text { for all } 1 \leq s<q,
$$

and, in particular, $\left\{v_{n}\right\}$ still generates $\nu$. Moreover, if $\Omega=Q$ then $v_{n}-V \in L_{1 \text {-per }}^{q}\left(\mathbb{R}^{N} ; \mathbb{R}^{d}\right) \cap$ ker $\mathcal{A}$. 
(ii) $(q=1)$ Let $\left\{V_{n}\right\}$ be a sequence converging weakly in $L^{1}\left(\Omega ; \mathbb{R}^{d}\right)$ to a function $V, \mathcal{A} V_{n} \rightarrow 0$ in $W^{-1, r}\left(\Omega ; \mathbb{R}^{l}\right)$ for some $r \in(1, N /(N-1))$, and assume that $\left\{V_{n}\right\}$ generates a Young measure $\nu$. Then there exists an equi-integrable sequence $\left\{v_{n}\right\} \subset L^{1}\left(\Omega ; \mathbb{R}^{d}\right) \cap \operatorname{ker} \mathcal{A}$ such that

$$
\int_{\Omega} v_{n} d x=\int_{\Omega} V d x, \quad\left\|v_{n}-V_{n}\right\|_{L^{1}(\Omega)} \rightarrow 0
$$

and, in particular, $\left\{v_{n}\right\}$ still generates $\nu$. Moreover, if $\Omega=Q$ then $v_{n}-V \in L_{1-\mathrm{per}}^{1}\left(\mathbb{R}^{N} ; \mathbb{R}^{d}\right) \cap \operatorname{ker} \mathcal{A}$.

(iii) $(q=+\infty)$ Let $\left\{V_{n}\right\}$ be a sequence that satisfies $V_{n} \stackrel{\star}{\rightarrow} V$ in $L^{\infty}\left(\Omega ; \mathbb{R}^{d}\right), \mathcal{A} V_{n} \rightarrow 0$ in $L^{r}(\Omega)$ for some $r>N$, and assume that $\left\{V_{n}\right\}$ generates a Young measure $\nu$. Then there exists a sequence $\left\{v_{n}\right\} \subset L^{\infty}\left(\Omega ; \mathbb{R}^{d}\right) \cap \operatorname{ker} \mathcal{A}$ such that

$$
\int_{\Omega} v_{n} d x=\int_{\Omega} V d x, \quad\left\|v_{n}-V_{n}\right\|_{L^{\infty}(\Omega)} \rightarrow 0
$$

and, in particular, $\left\{v_{n}\right\}$ still generates $\nu$. Moreover, if $\Omega=Q$ then $v_{n}-V \in L_{1 \text {-per }}^{\infty}\left(\mathbb{R}^{N} ; \mathbb{R}^{d}\right) \cap \operatorname{ker} \mathcal{A}$.

In the second part of the paper we will need the following classical results from $\Gamma$-convergence. For a proof see $[10]$.

Proposition 2.4. Let $(X, d)$ be a separable metric space and let $f_{n}: X \rightarrow[-\infty, \infty]$. Then

(i) there exists an increasing sequence of integers $\left\{n_{k}\right\}$ such that

$$
\Gamma(d)-\lim _{k \rightarrow \infty} f_{n_{k}}(x) \quad \text { exists for all } x \in X .
$$

(ii) Moreover

$$
f_{\infty}=\Gamma(d)-\lim _{n \rightarrow \infty} f_{n}
$$

if and only if for every subsequence $\left\{f_{n_{k}}\right\}$ there exists a further subsequence $\left\{f_{n_{k_{j}}}\right\}$ which $\Gamma(d)$-converges to $f_{\infty}$.

\section{RELAXATION}

In this section we prove Theorem 1.1 and its generalization to the case where $q \in\{1, \infty\}$ and $p=\infty$ (see Th. 3.6).

Lemma 3.1. Let $f: \Omega \times \mathbb{R}^{m} \times \mathbb{R}^{d} \rightarrow[0, \infty)$ be a Carathéodory function satisfying $(H)$, with $1 \leq p<\infty$ and $1<q<\infty$. Let $(u, v) \in L^{p}\left(D ; \mathbb{R}^{m}\right) \times\left(L^{q}\left(D ; \mathbb{R}^{d}\right) \cap \operatorname{ker} \mathcal{A}\right)$, where $D \in \mathcal{O}(\Omega)$, and consider a sequence of functions $\left\{\left(u_{k}, \hat{v}_{k}\right)\right\} \subset L^{p}\left(D ; \mathbb{R}^{m}\right) \times L^{q}\left(D ; \mathbb{R}^{d}\right)$ such that

$$
\begin{gathered}
u_{k} \rightarrow u \quad \text { in } \quad L^{p}\left(D ; \mathbb{R}^{m}\right), \quad \hat{v}_{k} \rightarrow v \quad \text { in } \quad L^{q}\left(D ; \mathbb{R}^{d}\right) \\
\mathcal{A} \hat{v}_{k} \rightarrow 0 \quad \text { in } W^{-1, q}\left(D ; \mathbb{R}^{l}\right) .
\end{gathered}
$$

Then we can find a q-equi-integrable sequence $\left\{v_{k}\right\} \subset L^{q}\left(D ; \mathbb{R}^{d}\right) \cap \operatorname{ker} \mathcal{A}$ such that

$$
v_{k} \rightarrow v \quad \text { in } \quad L^{q}\left(D ; \mathbb{R}^{d}\right), \quad \int_{D} v_{k} d x=\int_{D} v d x
$$

and

$$
\liminf _{k \rightarrow \infty} \int_{D} f\left(x, u(x), v_{k}(x)\right) d x \leq \liminf _{k \rightarrow \infty} \int_{D} f\left(x, u_{k}(x), \hat{v}_{k}(x)\right) d x .
$$


Proof of Lemma 3.1. Consider a subsequence $\left\{\left(u_{n}, \hat{v}_{n}\right)\right\}$ of $\left\{\left(u_{k}, \hat{v}_{k}\right)\right\}$ such that

$$
\lim _{n \rightarrow \infty} \int_{D} f\left(x, u_{n}(x), \hat{v}_{n}(x)\right) d x=\liminf _{k \rightarrow \infty} \int_{D} f\left(x, u_{k}(x), \hat{v}_{k}(x)\right) d x
$$

and $\left\{\left(u_{n}, \hat{v}_{n}\right)\right\}$ generates the Young measure $\left\{\delta_{u(x)} \otimes \nu_{x}\right\}_{x \in D}$. For $i \in \mathbb{N}$ let

$$
F_{i}:=\left\{x \in D: \operatorname{dist}(x, \partial D)<\frac{1}{i}\right\}
$$

and consider cut-off functions $\theta_{i}$ with compact support in $D$ and such that $\theta_{i} \equiv 1$ in $D \backslash F_{i}$. Set $w_{i, n}:=$ $\theta_{i}\left(\hat{v}_{n}-v\right) \in L^{q}\left(D ; \mathbb{R}^{d}\right)$ and fix $\varphi \in L^{q^{\prime}}\left(D ; \mathbb{R}^{d}\right)$, where $q^{\prime}$ is the Hölder conjugate exponent of $q$. Then

$$
\lim _{i \rightarrow \infty} \lim _{n \rightarrow \infty} \int_{D} \varphi(x) w_{i, n}(x) d x=\lim _{i \rightarrow \infty} \lim _{n \rightarrow \infty} \int_{D} \varphi(x) \theta_{i}(x)\left(\hat{v}_{n}(x)-v(x)\right) d x=0,
$$

where we have used the fact that $\hat{v}_{k} \rightarrow v$ in $L^{q}\left(D ; \mathbb{R}^{d}\right)$. Hence $w_{i, n} \rightarrow 0$ in $L^{q}\left(D ; \mathbb{R}^{d}\right)$ as $n \rightarrow \infty$ and $i \rightarrow \infty$. Moreover, in view of the compact embedding

$$
L^{q}\left(D ; \mathbb{R}^{l}\right) \hookrightarrow W^{-1, q}\left(D ; \mathbb{R}^{l}\right)
$$

and the assumption that $\mathcal{A} \hat{v}_{k} \rightarrow 0$ in $W^{-1, q}\left(D ; \mathbb{R}^{l}\right)$, we have that

$$
\lim _{i \rightarrow \infty} \lim _{n \rightarrow \infty} \mathcal{A} w_{i, n}=0 \quad \text { in } \quad W^{-1, q}\left(D ; \mathbb{R}^{l}\right) .
$$

Let $\mathbb{G}$ be a countable dense subset of $L^{q}\left(D ; \mathbb{R}^{d}\right)$. By means of a diagonalization process we obtain subsequences $\left\{u_{i}:=u_{n_{i}}\right\}$ and $\left\{\hat{w}_{i}:=w_{i, n_{i}}=\theta_{i}\left(\hat{v}_{n_{i}}-v\right)\right\}$ such that $\left\|u_{i}-u\right\|_{L^{p}} \rightarrow 0,(3.2)$ holds for each $\varphi \in \mathbb{G}$, and

$$
\mathcal{A} \hat{w}_{i} \rightarrow 0 \quad \text { in } \quad W^{-1, q}\left(D ; \mathbb{R}^{l}\right) .
$$

Hence $\hat{w}_{i} \rightarrow 0$ in $L^{q}\left(D ; \mathbb{R}^{d}\right)$, by the density of $\mathbb{G}$ in $L^{q}\left(D ; \mathbb{R}^{d}\right)$. By Proposition 2.3(i) there exists a $q$-equiintegrable sequence $\left\{w_{i}\right\} \subset L^{q}\left(D ; \mathbb{R}^{d}\right) \cap \operatorname{ker} \mathcal{A}$ such that $w_{i} \rightarrow 0$ in $L^{q}\left(D ; \mathbb{R}^{d}\right)$, and

$$
\int_{D} w_{i} d x=0, \quad\left\|\hat{w}_{i}-w_{i}\right\|_{L^{s}(D)} \rightarrow 0 \quad \text { for all } 1 \leq s<q .
$$

Set $v_{i}:=v+w_{i}$. Then $\int_{D} v_{i} d x=\int_{D} v d x, v_{i} \rightarrow v$ in $L^{q}\left(D ; \mathbb{R}^{d}\right)$. By Hölder's inequality and by (3.3), for $1 \leq s<q$

$$
\begin{aligned}
\left\|\hat{v}_{n_{i}}-v_{i}\right\|_{L^{s}(D)} & \leq\left\|\hat{v}_{n_{i}}-v-\hat{w}_{i}\right\|_{L^{s}(D)}+\left\|\hat{w}_{i}-w_{i}\right\|_{L^{s}(D)} \\
& \leq\left\|\left(1-\theta_{i}\right)\left(\hat{v}_{n_{i}}-v\right)\right\|_{L^{s}(D)}+\left\|\hat{w}_{i}-w_{i}\right\|_{L^{s}(D)} \\
& \leq\left\|\hat{v}_{n_{i}}-v\right\|_{L^{q}(D)}\left|F_{i}\right|^{r}+\left\|\hat{w}_{i}-w_{i}\right\|_{L^{s}(D)} \rightarrow 0
\end{aligned}
$$

as $i \rightarrow \infty$ and where $r:=(q-s) / s q$. By (3.4) and Proposition 2.2, the two sequences

$$
\left\{\left(u(x), v_{i}(x)\right)\right\} \quad \text { and } \quad\left\{\left(u_{i}(x), \hat{v}_{i}(x)\right)\right\}
$$

generate the same Young measure $\left\{\delta_{u(x)} \otimes \nu_{x}\right\}_{x \in D}$. Hence by Theorems 2.1(ii) and (iii)

$$
\begin{aligned}
\lim _{i \rightarrow \infty} \int_{D} f\left(x, u(x), v_{i}(x)\right) d x & =\int_{D} \int_{\mathbb{R}^{d}} f(x, u(x), V) d \nu_{x}(V) d x \leq \liminf _{i \rightarrow \infty} \int_{D} f\left(x, u_{i}(x), \hat{v}_{i}(x)\right) d x \\
& =\liminf _{k \rightarrow \infty} \int_{D} f\left(x, u_{k}(x), \hat{v}_{k}(x)\right) d x
\end{aligned}
$$


where we have used the fact that $\left\{f\left(x, u(x), v_{i}(x)\right)\right\}$ is equi-integrable over $D$, which follows from $(H)$ and the $q$-equi-integrability of $\left\{v_{i}\right\}$ over $D$.

It follows immediately from Lemma 3.1 that under its assumptions on $f$ it holds:

Corollary 3.2. For $D \in \mathcal{O}(\Omega)$ and $(u, v) \in L^{p}\left(\Omega ; \mathbb{R}^{m}\right) \times\left(L^{q}\left(\Omega ; \mathbb{R}^{d}\right) \cap \operatorname{ker} \mathcal{A}\right)$

$$
\begin{gathered}
\mathcal{F}((u, v) ; D)=\inf \left\{\liminf _{n \rightarrow \infty} \int_{D} g\left(x, v_{n}(x)\right) d x:\left\{v_{n}\right\} \subset L^{q}\left(D ; \mathbb{R}^{d}\right) \cap \operatorname{ker} \mathcal{A}\right. \text { is q-equi-integrable } \\
\text { and } \left.v_{n} \rightarrow v \text { in } L^{q}\left(D ; \mathbb{R}^{d}\right)\right\},
\end{gathered}
$$

where $g$ is the Carathéodory function defined by

$$
g(x, v):=f(x, u(x), v)
$$

Note that, by $(H)$, the function $g$ satisfies the growth condition

$$
0 \leq g(x, v) \leq C\left(1+|u(x)|^{p}+|v|^{q}\right)
$$

for a.e. $x \in \Omega$ and all $v \in \mathbb{R}^{d}$. Moreover, since $g$ is a Carathéodory function, by the Scorza-Dragoni theorem for each $j \in \mathbb{N}$ there exists a compact set $K_{j} \subset \Omega$, with $\left|\Omega \backslash K_{j}\right| \leq 1 / j$, such that $g: K_{j} \times \mathbb{R}^{d} \rightarrow[0, \infty)$ is continuous. Let $K_{j}^{*}$ be the set of Lebesgue points of $\chi_{K_{j}}$, and set

$$
\omega:=\bigcup_{j}^{\infty}\left(K_{j} \cap K_{j}^{*}\right) \cap L(u, v)
$$

where $L(u, v)$ is the set of Lebesgue points of $(u, v)$. Then

$$
|\Omega \backslash \omega| \leq\left|\Omega \backslash K_{j}\right| \leq \frac{1}{j} \rightarrow 0 \quad \text { as } j \rightarrow \infty .
$$

Corollary 3.3. Assume that $x_{0} \in \omega$, let $v \in L^{q}\left(Q ; \mathbb{R}^{d}\right) \cap \operatorname{ker} \mathcal{A}$, and consider $r_{k} \rightarrow 0^{+}$and a sequence of functions

$$
\left\{\hat{v}_{k}\right\} \subset L^{q}\left(Q ; \mathbb{R}^{d}\right) \cap \operatorname{ker} \mathcal{A}
$$

such that

$$
\hat{v}_{k} \rightarrow v \quad \text { in } \quad L^{q}\left(Q ; \mathbb{R}^{d}\right) .
$$

Then we can find a q-equi-integrable sequence $\left\{w_{k}\right\} \subset L_{1-\text { per }}^{q}\left(\mathbb{R}^{N} ; \mathbb{R}^{d}\right) \cap \operatorname{ker} \mathcal{A}$ such that

$$
w_{k} \rightarrow 0 \quad \text { in } \quad L^{q}\left(Q ; \mathbb{R}^{d}\right), \quad \int_{Q} w_{k} d x=0
$$

and

$$
\liminf _{k \rightarrow \infty} \int_{Q} g\left(x_{0}, v(y)+w_{k}(y)\right) d y \leq \liminf _{k \rightarrow \infty} \int_{Q} g\left(x_{0}+r_{k} y, \hat{v}_{k}(y)\right) d y
$$


Proof of Corollary 3.3. We proceed as in the proof of Lemma 3.1 up to (3.4). Since the sequence $\left\{v_{i}\right\}$ is $q$-equiintegrable, for any $\eta>0$ there exists $\delta>0$ such that

$$
\sup _{i} \int_{D} C\left(1+\left|u\left(x_{0}\right)\right|^{p}+\left|v_{i}(y)\right|^{q}\right) d y<\eta
$$

for any measurable set $D \subset Q$, with $|D|<\delta$, and where $C$ is the constant given in $(H)$. Fix $\eta>0$ and let $\delta>0$ be given according to (3.8). By the Biting Lemma (see [6]) we may find a further subsequence $\left\{\hat{v}_{n_{j}}\right\} \subset\left\{\hat{v}_{n_{i}}\right\}$ and a set $E \subset Q$ such that $|Q \backslash E|<\delta$ and $\left\{\hat{v}_{n_{j}}\right\}$ is $q$-equi-integrable over $E$. Hence there exists $0<\delta_{1}<\delta$ such that

$$
\sup _{j} \int_{D} C\left(1+\left|u\left(x_{0}\right)\right|^{p}+\left|\hat{v}_{n_{j}}(y)\right|^{q}\right) d y<\eta
$$

for any measurable set $D \subset E$, with $|D|<\delta_{1}$. Moreover, as $\left\{\hat{v}_{n_{j}}\right\},\left\{v_{j}\right\}$ are bounded in $L^{q}\left(Q ; \mathbb{R}^{d}\right)$, we may find $L>0$ such that

$$
\left|E \backslash E_{j}\right| \leq \delta_{1}, \quad \text { where } \quad E_{j}:=\left\{y \in E:\left|\hat{v}_{n_{j}}(y)\right| \leq L,\left|v_{j}(y)\right| \leq L\right\} .
$$

Note that by construction of $v_{i}$ and by Proposition $2.3, v_{i}=v+w_{i}$ where $w_{i} \in L_{1-\text { per }}^{q}\left(\mathbb{R}^{N} ; \mathbb{R}^{d}\right) \cap$ ker $\mathcal{A}$. From the definition of the set $\omega$ there exists an integer $j_{0}$ such that $x_{0} \in K_{j_{0}} \cap K_{j_{0}}^{*}$. Since

$$
g: K_{j_{0}} \times \overline{B_{d}(0, L)} \rightarrow[0, \infty)
$$

is uniformly continuous, there exists $\rho>0$ such that

$$
\left|g(x, v)-g\left(x_{1}, v\right)\right| \leq \eta
$$

for all $(x, v),\left(x_{1}, v\right) \in K_{j_{0}} \times \overline{B_{d}(0, L)}$, with $\left|x-x_{1}\right| \leq \rho$. By (3.10) and (3.11)

$$
\begin{aligned}
\lim _{i \rightarrow \infty} \int_{Q} g\left(x_{0}+r_{n_{i}} y, \hat{v}_{n_{i}}(y)\right) d y & \geq \liminf _{j \rightarrow \infty} \int_{E_{j}} g\left(x_{0}+r_{n_{j}} y, \hat{v}_{n_{j}}(y)\right) d y \\
& \geq \liminf _{j \rightarrow \infty} \frac{1}{r_{n_{j}}^{N}} \int_{\left(x_{0}+r_{n_{j}} E_{j}\right) \cap K_{j_{0}}} g\left(x, \hat{v}_{n_{j}}\left(\left(x-x_{0}\right) / r_{n_{j}}\right)\right) d x \\
& \geq-\eta+\liminf _{j \rightarrow \infty} \frac{1}{r_{n_{j}}^{N}} \int_{\left(x_{0}+r_{n_{j}} E_{j}\right) \cap K_{j_{0}}} g\left(x_{0}, \hat{v}_{n_{j}}\left(\left(x-x_{0}\right) / r_{n_{j}}\right)\right) d x .
\end{aligned}
$$

Using, once again, the fact that $\left|\hat{v}_{n_{j}}(y)\right| \leq L$ for $y \in E_{j}$, by (3.6) we have that

$$
\frac{1}{r_{n_{j}}^{N}} \int_{\left(x_{0}+r_{n_{j}} E_{j}\right) \backslash K_{j_{0}}} g\left(x_{0}, \hat{v}_{n_{j}}\left(\left(x-x_{0}\right) / r_{n_{j}}\right)\right) d x \leq C\left(1+\left|u\left(x_{0}\right)\right|^{p}+L^{q}\right) \frac{\left|Q\left(x_{0}, r_{n_{j}}\right) \backslash K_{j_{0}}\right|}{r_{n_{j}}^{N}} \rightarrow 0
$$

as $j \rightarrow \infty$, because $x_{0}$ is a Lebesgue point of $\chi_{K_{j_{0}}}$. Consequently, from (3.12) we get

$$
\begin{aligned}
\lim _{i \rightarrow \infty} \int_{Q} g\left(x_{0}+r_{n_{i}} y, \hat{v}_{n_{i}}(y)\right) d y & \geq-\eta+\liminf _{j \rightarrow \infty} \frac{1}{r_{n_{j}}^{N}} \int_{x_{0}+r_{n_{j}} E_{j}} g\left(x_{0}, \hat{v}_{n_{j}}\left(\left(x-x_{0}\right) / r_{n_{j}}\right)\right) d x \\
& =-\eta+\liminf _{j \rightarrow \infty} \int_{E_{j}} g\left(x_{0}, \hat{v}_{n_{j}}(y)\right) d y \\
& \geq-2 \eta+\liminf _{j \rightarrow \infty} \int_{E} g\left(x_{0}, \hat{v}_{n_{j}}(y)\right) d y,
\end{aligned}
$$


where we have used $(3.6,3.9)$ and the fact that $\left|E \backslash E_{j}\right| \leq \delta_{1}$. We may now proceed as in the previous lemma, using the Carathéory function $h(x, v):=\chi_{E}(x) g\left(x_{0}, v\right)$, to obtain

$$
\lim _{i \rightarrow \infty} \int_{Q} g\left(x_{0}+r_{n_{i}} y, \hat{v}_{n_{i}}(y)\right) d y \geq-2 \eta+\liminf _{j \rightarrow \infty} \int_{E} g\left(x_{0}, v_{j}(y)\right) d y \geq-3 \eta+\liminf _{j \rightarrow \infty} \int_{Q} g\left(x_{0}, v_{j}(y)\right) d y
$$

by (3.8). It now suffices to let $\eta \rightarrow 0^{+}$.

Theorem 1.1 follows from Lemmas 3.4 and 3.5 below. We will use the notation $\mu\lfloor A$ to denote the restriction of a Radon measure $\mu$ to the Borel set A, i.e., $\mu\lfloor A(X):=\mu(X \cap A)$ where $X$ is an arbitrary Borel set in the domain of $\mu$.

Lemma 3.4. $\mathcal{F}((u, v) ; \cdot)$ is the trace of a Radon measure absolutely continuous with respect to $\mathcal{L}^{N}\lfloor\Omega$.

Proof of Lemma 3.4. As it is usual, it suffices to prove subadditivity (see e.g. [3, 21]), i.e.

$$
\mathcal{F}((u, v) ; D) \leq \mathcal{F}((u, v) ; D \backslash \bar{B})+\mathcal{F}((u, v) ; C)
$$

if $B \subset \subset C \subset \subset D$. Fix $\eta>0$. By Corollary 3.2 there exist two $q$-equi-integrable sequences

$$
\left\{v_{k}\right\} \subset L^{q}\left(D \backslash \bar{B} ; \mathbb{R}^{d}\right) \cap \operatorname{ker} \mathcal{A}, \quad\left\{w_{k}\right\} \subset L^{q}\left(C ; \mathbb{R}^{d}\right) \cap \operatorname{ker} \mathcal{A},
$$

such that

$$
v_{k} \rightarrow v \quad \text { in } \quad L^{q}\left(D \backslash \bar{B} ; \mathbb{R}^{d}\right), \quad w_{k} \rightarrow v \quad \text { in } \quad L^{q}\left(C ; \mathbb{R}^{d}\right)
$$

and

$$
\begin{aligned}
& \lim _{k \rightarrow \infty} \int_{D \backslash \bar{B}} g\left(x, v_{k}(x)\right) d x \leq \mathcal{F}((u, v) ; D \backslash \bar{B})+\eta, \\
& \lim _{k \rightarrow \infty} \int_{C} g\left(x, w_{k}(x)\right) d x \leq \mathcal{F}((u, v) ; C)+\eta .
\end{aligned}
$$

Let $\theta_{j}$ be smooth cut-off functions, $\theta_{j} \in C_{c}^{\infty}(C ;[0,1]), \theta_{j}(x)=1$ for all $x \in B$, and $\left|\left\{0<\theta_{j}<1\right\}\right| \rightarrow 0$ as $j \rightarrow \infty$. Set

$$
\hat{V}_{j, k}:=\left(1-\theta_{j}\right) v_{k}+\theta_{j} w_{k}
$$

Then, for $j$ fixed,

$$
\mathcal{A} \hat{V}_{j, k}=\left(1-\theta_{j}\right) \mathcal{A} v_{k}+\theta_{j} \mathcal{A} w_{k}-\sum_{i=1}^{N} A^{(i)} v_{k} \frac{\partial \theta_{j}}{\partial x_{i}}+\sum_{i=1}^{N} A^{(i)} w_{k} \frac{\partial \theta_{j}}{\partial x_{i}} \rightarrow 0
$$

as $k \rightarrow \infty$ in $W^{-1, q}\left(D ; \mathbb{R}^{l}\right)$ strong. Using a diagonalization procedure such as that adopted in the proof of Lemma 3.1, we get

$$
\hat{V}_{j} \rightarrow v \quad \text { in } \quad L^{q}\left(D ; \mathbb{R}^{d}\right), \quad \mathcal{A} \hat{V}_{j} \rightarrow 0 \quad \text { in } \quad W^{-1, q}\left(D ; \mathbb{R}^{l}\right),
$$

where $\hat{V}_{j}:=\hat{V}_{j, k_{j}}$. By Lemma 3.1 we can find a $q$-equi-integrable sequence $\left\{V_{j}\right\} \subset L^{q}\left(D ; \mathbb{R}^{d}\right) \cap \operatorname{ker} \mathcal{A}$ such that $V_{j} \rightarrow v$ in $L^{q}\left(D ; \mathbb{R}^{d}\right)$ and

$$
\liminf _{j \rightarrow \infty} \int_{D} g\left(x, V_{j}(x)\right) d x \leq \liminf _{j \rightarrow \infty} \int_{D} g\left(x, \hat{V}_{j}(x)\right) d x
$$


Consequently, in view of Corollary 3.2

$$
\begin{aligned}
\mathcal{F}((u, v) ; D) \leq & \liminf _{j \rightarrow \infty} \int_{D} g\left(x, V_{j}(x)\right) d x \leq \liminf _{j \rightarrow \infty} \int_{D} g\left(x, \hat{V}_{j}(x)\right) d x \\
\leq & \limsup _{j \rightarrow \infty} \int_{\left\{\theta_{j}=0\right\}} g\left(x, v_{k_{j}}(x)\right) d x+\limsup _{j \rightarrow \infty} \int_{\left\{\theta_{j}=1\right\}} g\left(x, w_{k_{j}}(x)\right) d x \\
& +\limsup _{j \rightarrow \infty} \int_{\left\{0<\theta_{j}<1\right\}} C\left(1+|u(x)|^{p}+\left|w_{k_{j}}(x)\right|^{q}+\left|v_{k_{j}}(x)\right|^{q}\right) d x \\
\leq & 2 \eta+\mathcal{F}((u, v) ; D \backslash \bar{B})+\mathcal{F}((u, v) ; C) .
\end{aligned}
$$

It suffices to let $\eta \rightarrow 0^{+}$. Finally, note that by $(H)$ we have that

$$
\mathcal{F}((u, v), \cdot) \leq C\left(1+|u|^{p}+|v|^{q}\right) \mathcal{L}^{N}\lfloor\Omega
$$

Lemma 3.5. For $\mathcal{L}^{N}$ a.e. $x_{0} \in \Omega$ we have

$$
\frac{d \mathcal{F}((u, v) ; \cdot)}{d \mathcal{L}^{N}}\left(x_{0}\right)=\mathcal{Q}_{\mathcal{A}} f\left(x_{0}, u\left(x_{0}\right), v\left(x_{0}\right)\right)
$$

Proof of Lemma 3.5. Fix $x_{0} \in \omega$, where $\omega$ is defined as in (3.7), and such that

$$
\lim _{r \rightarrow 0^{+}} \frac{1}{r^{N}} \int_{Q\left(x_{0}, r\right)}\left|u(x)-u\left(x_{0}\right)\right|^{p} d x=\lim _{r \rightarrow 0^{+}} \frac{1}{r^{N}} \int_{Q\left(x_{0}, r\right)}\left|v(x)-v\left(x_{0}\right)\right|^{q} d x=0
$$

and

$$
\frac{d \mathcal{F}((u, v) ; \cdot)}{d \mathcal{L}^{N}}\left(x_{0}\right)=\lim _{r \rightarrow 0^{+}} \frac{\mathcal{F}\left((u, v) ; Q\left(x_{0}, r\right)\right)}{r^{N}}<\infty
$$

where, by virtue of Lemma 3.4, we have chosen the radii $r \rightarrow 0^{+}$such that

$$
\mathcal{F}\left((u, v) ; \partial\left(Q\left(x_{0}, r\right)\right)\right)=0
$$

By Corollary 3.2 and for $r>0$ fixed, let $\left\{v_{n, r}\right\} \subset L^{q}\left(Q\left(x_{0}, r\right) ; \mathbb{R}^{d}\right) \cap$ ker $\mathcal{A}$ be such that $v_{n, r} \rightarrow v$ in $L^{q}\left(Q\left(x_{0}, r\right) ; \mathbb{R}^{d}\right)$ as $n \rightarrow \infty$ and

$$
\lim _{n \rightarrow \infty} \int_{Q\left(x_{0}, r\right)} g\left(x, v_{n, r}(x)\right) d x \leq \mathcal{F}\left((u, v) ; Q\left(x_{0}, r\right)\right)+r^{N+1}
$$

Then

$$
\frac{d \mathcal{F}((u, v) ; \cdot)}{d \mathcal{L}^{N}}\left(x_{0}\right) \geq \liminf _{r \rightarrow 0^{+}} \lim _{n \rightarrow \infty} \frac{1}{r^{N}} \int_{Q\left(x_{0}, r\right)} g\left(x, v_{n . r}(x)\right) d x=\liminf _{r \rightarrow 0^{+}} \lim _{n \rightarrow \infty} \int_{Q} g\left(x_{0}+r y, v\left(x_{0}\right)+w_{n, r}(y)\right) d y
$$

where $w_{n, r}(y):=v_{n, r}\left(x_{0}+r y\right)-v\left(x_{0}\right)$. We claim that $w_{n, r} \rightarrow 0$ in $L^{q}\left(Q ; \mathbb{R}^{d}\right)$ if we first let $n \rightarrow \infty$ and then $r \rightarrow 0^{+}$. Indeed let $\varphi \in L^{q^{\prime}}\left(Q ; \mathbb{R}^{d}\right)$, where $q^{\prime}$ is the Hölder conjugate exponent of $q$. Using Hölder's inequality 
and then making a change of variables, we get

$$
\begin{aligned}
\left|\int_{Q} \varphi(y) w_{r, n}(y) d y\right| \leq & \left|\int_{Q} \varphi(y)\left(v_{n, r}\left(x_{0}+r y\right)-v\left(x_{0}+r y\right)\right) d y\right|+\left|\int_{Q} \varphi(y)\left(v\left(x_{0}+r y\right)-v\left(x_{0}\right)\right) d y\right| \\
\leq & \left|\frac{1}{r^{N}} \int_{Q\left(x_{0}, r\right)} \varphi\left(\left(x-x_{0}\right) / r\right)\left(v_{n, r}(x)-v(x)\right) d x\right| \\
& +\|\varphi\|_{L^{q^{\prime}}(Q)}\left(\frac{1}{r^{N}} \int_{Q\left(x_{0}, r\right)}\left|v(x)-v\left(x_{0}\right)\right|^{q} d x\right)^{1 / q}
\end{aligned}
$$

If we now let $n \rightarrow \infty$ the first integral tends to zero, since $v_{n, r} \rightarrow v$ in $L^{q}\left(Q\left(x_{0}, r\right) ; \mathbb{R}^{d}\right)$. The claim then follows by letting $r \rightarrow 0^{+}$and by using (3.13). Diagonalize to get $\hat{w}_{k} \in L^{q}\left(Q ; \mathbb{R}^{d}\right) \cap$ ker $\mathcal{A}$ such that $\hat{w}_{k} \rightarrow 0$ in $L^{q}\left(Q ; \mathbb{R}^{d}\right)$ and

$$
\frac{d \mathcal{F}((u, v) ; \cdot)}{d \mathcal{L}^{N}}\left(x_{0}\right) \geq \lim _{k \rightarrow \infty} \int_{Q} g\left(x_{0}+r_{k} y, v\left(x_{0}\right)+\hat{w}_{k}(y)\right) d y
$$

where $r_{k} \rightarrow 0$. By Corollary 3.3 there is a $q$-equi-integrable sequence $\left\{w_{k}\right\} \subset L_{1 \text {-per }}^{q}\left(\mathbb{R}^{N} ; \mathbb{R}^{d}\right) \cap$ ker $\mathcal{A}$ such that

$$
w_{k} \rightarrow 0 \quad \text { in } \quad L^{q}\left(Q ; \mathbb{R}^{d}\right), \quad \int_{Q} w_{k} d y=0
$$

and

$$
\begin{aligned}
\frac{d \mathcal{F}((u, v) ; \cdot)}{d \mathcal{L}^{N}}\left(x_{0}\right) & \geq \lim _{k \rightarrow \infty} \int_{Q} g\left(x_{0}+r_{k} y, v\left(x_{0}\right)+\hat{w}_{k}(y)\right) d y \\
& \geq \liminf _{k \rightarrow \infty} \int_{Q} f\left(x_{0}, u\left(x_{0}\right), v\left(x_{0}\right)+w_{k}(y)\right) d y \geq \mathcal{Q}_{\mathcal{A}} f\left(x_{0}, u\left(x_{0}\right), v\left(x_{0}\right)\right) .
\end{aligned}
$$

To conclude the proof of the lemma it remains to show that

$$
\frac{d \mathcal{F}((u, v) ; \cdot)}{d \mathcal{L}^{N}}\left(x_{0}\right) \leq \mathcal{Q}_{\mathcal{A}} f\left(x_{0}, u\left(x_{0}\right), v\left(x_{0}\right)\right) \quad \text { for } \mathcal{L}^{N} \text { a.e. } x_{0} \in \Omega
$$

Fix $\eta>0$ and let $w \in C_{1-\text { per }}^{\infty}\left(\mathbb{R}^{N} ; \mathbb{R}^{d}\right) \cap \operatorname{ker} \mathcal{A}$ be such that $\int_{Q} w d y=0$ and

$$
\int_{Q} f\left(x_{0}, u\left(x_{0}\right), v\left(x_{0}\right)+w(y)\right) d y \leq \mathcal{Q}_{\mathcal{A}} f\left(x_{0}, u\left(x_{0}\right), v\left(x_{0}\right)\right)+\eta .
$$

For any fixed $r>0$ set $w_{n, r}(x):=w\left(n\left(x-x_{0}\right) / r\right)$. Then $w_{n, r} \rightarrow 0$ in $L^{q}\left(Q\left(x_{0}, r\right) ; \mathbb{R}^{d}\right)$ as $n \rightarrow \infty$. Hence, by Corollary 3.2,

$$
\frac{d \mathcal{F}((u, v) ; \cdot)}{d \mathcal{L}^{N}}\left(x_{0}\right)=\lim _{r \rightarrow 0^{+}} \frac{\mathcal{F}\left((u, v) ; Q\left(x_{0}, r\right)\right)}{r^{N}} \leq \liminf _{r \rightarrow 0^{+}} \liminf _{n \rightarrow \infty} \frac{1}{r^{N}} \int_{Q\left(x_{0}, r\right)} g\left(x, v(x)+w_{n, r}(x)\right) d x .
$$

Fix $L>\left|v\left(x_{0}\right)\right|+\left.|| w\right|_{L^{\infty}}+1$, and let $j$ be such that $x_{0} \in K_{j} \cap K_{j}^{*} \cap L(u, v)$, where we are using the notation introduced in (3.7). Since

$$
g: K_{j} \times \overline{B_{d}(0, L)} \rightarrow[0, \infty)
$$

is uniformly continuous, there exists $0<\rho<1$ such that

$$
\left|g(x, v)-g\left(x_{1}, v_{1}\right)\right| \leq \eta
$$


for all $(x, v),\left(x_{1}, v_{1}\right) \in K_{j} \times \overline{B_{d}\left(v\left(x_{0}\right), L\right)}$, with $\left|x-x_{1}\right| \leq \rho$ and $\left|v-v_{1}\right| \leq \rho$. Let

$$
E_{r, \rho}:=\left\{x \in Q\left(x_{0}, r\right):\left|v(x)-v\left(x_{0}\right)\right| \leq \rho\right\} .
$$

We claim that

$$
\limsup _{r \rightarrow 0^{+}} \frac{1}{r^{N}} \int_{Q\left(x_{0}, r\right) \backslash\left(E_{r, \rho} \cap K_{j}\right)} C\left(1+|u(x)|^{p}+|v(x)|^{q}+\|w\|_{L^{\infty}}^{q}\right) d x=0 .
$$

Since $\left|v(x)-v\left(x_{0}\right)\right| \geq \rho$ for $x \in Q\left(x_{0}, r\right) \backslash E_{r, \rho}$, we have

$$
\begin{aligned}
\frac{1}{r^{N}} \int_{Q\left(x_{0}, r\right) \backslash\left(E_{r, \rho} \cap K_{j}\right)} C\left(1+|u(x)|^{p}+|v(x)|^{q}\right. & \left.+|| w||_{L^{\infty}}^{q}\right) d x \leq C \frac{\left|Q\left(x_{0}, r\right) \backslash\left(E_{r, \rho} \cap K_{j}\right)\right|}{r^{N}} \\
& +\frac{C}{r^{N}} \int_{Q\left(x_{0}, r\right)}\left(\left|u(x)-u\left(x_{0}\right)\right|^{p}+\left|v(x)-v\left(x_{0}\right)\right|^{q}\right) d x
\end{aligned}
$$

and

$$
\begin{aligned}
\frac{\left|Q\left(x_{0}, r\right) \backslash\left(E_{r, \rho} \cap K_{j}\right)\right|}{r^{N}} \leq \frac{\left|Q\left(x_{0}, r\right) \backslash K_{j}\right|}{r^{N}} & +\frac{\left|Q\left(x_{0}, r\right) \backslash E_{r, \rho}\right|}{r^{N}} \leq \frac{\left|Q\left(x_{0}, r\right) \backslash K_{j}\right|}{r^{N}} \\
& +\frac{1}{\rho^{q}} \frac{C}{r^{N}} \int_{Q\left(x_{0}, r\right)}\left|v(x)-v\left(x_{0}\right)\right|^{q} d x \rightarrow 0 \quad \text { as } r \rightarrow 0^{+},
\end{aligned}
$$

where we have used (3.13) and the fact that $x_{0}$ is a Lebesgue point of $\chi_{K_{j}}$. Then by (3.6, 3.15-3.17) and (3.14),

$$
\begin{aligned}
\frac{d \mathcal{F}((u, v) ; \cdot)}{d \mathcal{L}^{N}}\left(x_{0}\right) \leq & \liminf _{r \rightarrow 0^{+}} \liminf _{n \rightarrow \infty} \frac{1}{r^{N}} \int_{E_{r, \rho} \cap K_{j}} g\left(x, v(x)+w_{n, r}(x)\right) d x \\
& +\limsup _{r \rightarrow 0^{+}} \frac{1}{r^{N}} \int_{Q\left(x_{0}, r\right) \backslash\left(E_{r, \rho} \cap K_{j}\right)} C\left(1+|u(x)|^{p}+|v(x)|^{q}+\|w\|_{L^{\infty}}^{q}\right) d x \\
\leq & \eta+\liminf _{r \rightarrow 0^{+}} \liminf _{n \rightarrow \infty} \frac{1}{r^{N}} \int_{Q\left(x_{0}, r\right)} g\left(x_{0}, v\left(x_{0}\right)+w_{n, r}(x)\right) d x \\
= & \eta+\liminf _{n \rightarrow \infty} \int_{Q} g\left(x_{0}, v\left(x_{0}\right)+w(n y)\right) d y \\
= & \eta+\int_{Q} g\left(x_{0}, v\left(x_{0}\right)+w(y)\right) d y \leq 2 \eta+\mathcal{Q}_{\mathcal{A}} f\left(x_{0}, u\left(x_{0}\right), v\left(x_{0}\right)\right),
\end{aligned}
$$

by virtue of the equality

$$
\liminf _{n \rightarrow \infty} \int_{Q} g\left(x_{0}, v\left(x_{0}\right)+w(n y)\right) d x=\int_{Q} g\left(x_{0}, v\left(x_{0}\right)+w(y)\right) d y,
$$

which follows from the $Q$-periodicity of the function $g\left(x_{0}, v\left(x_{0}\right)+w(\cdot)\right)$. It now suffices to let $\eta \rightarrow 0^{+}$.

As mentioned in the introduction, Theorem 1.1 continues to hold when $q \in\{1, \infty\}$ and $p=\infty$. Indeed, let $1 \leq p, q \leq \infty$ and assume that

$\left(A_{4}\right) f: \Omega \times \mathbb{R}^{m} \times \mathbb{R}^{d} \rightarrow[0, \infty)$ is a Carathéodory function satisfying the following growth conditions for a.e. $x \in \Omega$ and all $(u, v) \in \mathbb{R}^{m} \times \mathbb{R}^{d}$ :

$$
0 \leq f(x, u, v) \leq C\left(1+|u|^{p}+|v|^{q}\right) \quad \text { if } 1 \leq p, q<\infty,
$$


where $C>0$;

$$
0 \leq f(x, u, v) \leq a(x, u)\left(1+|v|^{q}\right) \quad \text { if } p=\infty \text { and } 1 \leq q<\infty,
$$

where $\chi_{\Omega} a \in L_{\mathrm{loc}}^{\infty}\left(\mathbb{R}^{N} \times \mathbb{R}^{d} ;[0, \infty)\right)$;

$$
0 \leq f(x, u, v) \leq b(x, v)\left(1+|u|^{p}\right) \quad \text { if } 1 \leq p<\infty \text { and } q=\infty,
$$

where $\chi_{\Omega} b \in L_{\text {loc }}^{\infty}\left(\mathbb{R}^{N} \times \mathbb{R}^{m} ;[0, \infty)\right)$;

$$
\chi_{\Omega} f \in L_{\mathrm{loc}}^{\infty}\left(\mathbb{R}^{N} \times \mathbb{R}^{m} \times \mathbb{R}^{d} ;[0, \infty)\right) \quad \text { if } p=q=\infty .
$$

For $D \in \mathcal{O}(\Omega)$ and $(u, v) \in L^{p}\left(\Omega ; \mathbb{R}^{m}\right) \times\left(L^{q}\left(\Omega ; \mathbb{R}^{d}\right) \cap \operatorname{ker} \mathcal{A}\right)$ define

$$
\begin{aligned}
\mathcal{F}((u, v) ; D) & :=\inf \left\{\liminf _{n \rightarrow \infty} F\left(\left(u_{n}, v_{n}\right) ; D\right):\left(u_{n}, v_{n}\right) \in L^{p}\left(D ; \mathbb{R}^{m}\right) \times L^{1}\left(D ; \mathbb{R}^{d}\right),\right. \\
& \left.u_{n} \rightarrow u \text { in } L^{p}\left(D ; \mathbb{R}^{m}\right), \quad v_{n} \rightarrow v \text { in } L^{1}\left(D ; \mathbb{R}^{d}\right), \quad \mathcal{A} v_{n} \rightarrow 0 \quad \text { in } W^{-1, r}\left(D ; \mathbb{R}^{l}\right)\right\}
\end{aligned}
$$

if $q=1$ and for some $r \in(1, N /(N-1))$; as in (1.2), we set

$$
\begin{aligned}
\mathcal{F}((u, v) ; D) & :=\inf \left\{\liminf _{n \rightarrow \infty} F\left(\left(u_{n}, v_{n}\right) ; D\right):\left(u_{n}, v_{n}\right) \in L^{p}\left(D ; \mathbb{R}^{m}\right) \times L^{q}\left(D ; \mathbb{R}^{d}\right)\right. \\
& \left.u_{n} \rightarrow u \text { in } L^{p}\left(D ; \mathbb{R}^{m}\right), \quad v_{n} \rightarrow v \text { in } L^{q}\left(D ; \mathbb{R}^{d}\right), \quad \mathcal{A} v_{n} \rightarrow 0 \quad \text { in } W^{-1, q}\left(D ; \mathbb{R}^{l}\right)\right\}
\end{aligned}
$$

if $1<q<\infty$;

$$
\begin{aligned}
\mathcal{F}((u, v) ; D) & :=\inf \left\{\liminf _{n \rightarrow \infty} F\left(\left(u_{n}, v_{n}\right) ; D\right):\left(u_{n}, v_{n}\right) \in L^{p}\left(D ; \mathbb{R}^{m}\right) \times L^{\infty}\left(D ; \mathbb{R}^{d}\right),\right. \\
& \left.u_{n} \rightarrow u \operatorname{in} L^{p}\left(D ; \mathbb{R}^{m}\right), \quad v_{n} \stackrel{\star}{*} v \text { in } L^{\infty}\left(D ; \mathbb{R}^{d}\right), \quad \mathcal{A} v_{n} \rightarrow 0 \quad \text { in } L^{r}\left(D ; \mathbb{R}^{l}\right)\right\}
\end{aligned}
$$

if $q=\infty$ and for some $r>N$.

We can prove the following theorem:

Theorem 3.6. Under condition $\left(A_{4}\right)$ and the constant-rank hypothesis $(1.1)$, for all $D \in \mathcal{O}(\Omega), u \in L^{p}\left(\Omega ; \mathbb{R}^{m}\right)$ and $v \in L^{q}\left(\Omega ; \mathbb{R}^{d}\right) \cap \operatorname{ker} \mathcal{A}$, we have

$$
\mathcal{F}((u, v) ; D)=\int_{D} \mathcal{Q}_{\mathcal{A}} f(x, u(x), v(x)) d x
$$

Proof of Theorem 3.6.

Step 1. Assume first that $1 \leq p<\infty$ and $q=1$. The proof is similar to the one of Theorem 1.1, with the exceptions that in Lemma 3.1 condition (3.1) should be replaced by

$$
\begin{aligned}
u_{k} \rightarrow u \quad \text { in } \quad L^{p}\left(D ; \mathbb{R}^{m}\right), \quad \hat{v}_{k} \rightarrow v \quad \text { in } L^{1}\left(D ; \mathbb{R}^{d}\right), \\
\mathcal{A} \hat{v}_{n} \rightarrow 0 \quad \text { in } W^{-1, r}\left(D ; \mathbb{R}^{l}\right) \text { for some } r \in(1, N /(N-1)),
\end{aligned}
$$


that we use the compact embedding

$$
L^{1}\left(D ; \mathbb{R}^{l}\right) \hookrightarrow W^{-1, r}\left(D ; \mathbb{R}^{l}\right), \quad r \in(0, N /(N-1),
$$

to diagonalize $\left\{w_{i, n}\right\}$, and $(3.3,3.4)$ are replaced, respectively, by

$$
\begin{gathered}
\int_{D} w_{i} d x=0, \quad\left\|\hat{w}_{i}-w_{i}\right\|_{L^{1}(D)} \rightarrow 0, \\
\left\|\hat{v}_{n_{i}}-v_{i}\right\|_{L^{1}(D)} \leq\left\|\hat{v}_{n_{i}}-v-\hat{w}_{i}\right\|_{L^{1}(D)}+\left\|\hat{w}_{i}-w_{i}\right\|_{L^{1}(D)} \leq\left\|\left(1-\theta_{i}\right)\left(\hat{v}_{n_{i}}-v\right)\right\|_{L^{1}(D)}+\left\|\hat{w}_{i}-w_{i}\right\|_{L^{1}(D)} \\
\leq\left\|\hat{v}_{n_{i}}-v\right\|_{L^{1}\left(F_{i}\right)}+\left\|\hat{w}_{i}-w_{i}\right\|_{L^{1}(D)} \rightarrow 0,
\end{gathered}
$$

where we have used the fact that $\left\|\hat{v}_{n_{i}}-v\right\|_{L^{1}\left(F_{i}\right)} \rightarrow 0$ as $i \rightarrow \infty$, which is due to the equi-integrability of the original sequence $\left\{\hat{v}_{k}-v\right\}$ and the fact that $\left|F_{i}\right| \rightarrow 0$.

Step 2. If $p=\infty$ and $1 \leq q<\infty$ then in Lemma 3.1 the only change needed is in deriving (3.5), which now follows from the fact that, by (3),

$$
0 \leq f\left(x, u(x), v_{i}(x)\right) \leq A_{\infty}\left(1+\left|v_{i}(x)\right|^{q}\right),
$$

where $A_{\infty}:=\operatorname{esssup}\left\{a(x, u): x \in \Omega, \quad|u| \leq\|u\|_{\infty}\right\}<\infty$, and thus equi-integrability of $\left\{f\left(x, u, v_{i}\right)\right\}$ follows from the $q$-equi-integrability of $\left\{v_{i}\right\}$ over $D$. Moreover in the remaining of the proof of Theorem 1.1, the growth condition (3.6) should be replaced by

$$
0 \leq g(x, v) \leq A_{\infty}\left(1+|v|^{q}\right)
$$

for a.e. $x \in \Omega$ and all $v \in \mathbb{R}^{d}$.

Step 3. If $1 \leq p \leq \infty$ and $q=\infty$ then in Lemma 3.1 the hypothesis (3.1) should be replaced by

$$
\begin{gathered}
u_{k} \rightarrow u \quad \text { in } L^{p}\left(D ; \mathbb{R}^{m}\right), \quad \hat{v}_{k} \stackrel{\star}{\rightarrow} v \text { in } L^{\infty}\left(D ; \mathbb{R}^{d}\right), \\
A \hat{v}_{n} \rightarrow 0 \quad \text { in } L^{r}\left(D ; \mathbb{R}^{l}\right) \text { for some } r>N,
\end{gathered}
$$

the growth condition should be replaced by (3.19) if $1 \leq p<\infty, q=\infty$, and by $\chi_{\Omega} g \in L_{\mathrm{loc}}^{\infty}\left(\mathbb{R}^{N} \times \mathbb{R}^{d} ;[0, \infty)\right)$ if $p=q=\infty$, and we can proceed similarly to the proof of Lemma 3.1 to show that $w_{i, n} \stackrel{\star}{\rightarrow} 0$ in $L^{\infty}\left(D ; \mathbb{R}^{d}\right)$ and $\mathcal{A} w_{i, n} \rightarrow 0$ in $L^{r}\left(D ; \mathbb{R}^{l}\right)$, and use Proposition 2.3(iii) to get

$$
\left\|v_{i}-\hat{v}_{n_{i}}\right\|_{\infty} \rightarrow 0
$$

We omit the details.

\section{Proofs of Theorems 1.3 And 1.5}

Proof of Theorem 1.3. We present the proof for $1 \leq p<\infty$, the case $p=\infty$ being very similar. Fix $u \in$ $W^{s, p}\left(\Omega ; \mathbb{R}^{n}\right)$, and for $D \in \mathcal{O}(\Omega)$ define

$$
\begin{array}{r}
\mathcal{F}(u ; D):=\inf \left\{\liminf _{k \rightarrow \infty} \int_{D} f\left(x, u_{k}, \ldots, \nabla^{s} u_{k}\right) d x:\left\{u_{k}\right\} \subset W^{s, p}\left(D ; \mathbb{R}^{n}\right),\right. \\
\left.u_{k} \rightarrow u \text { in } W^{s, p}\left(D ; \mathbb{R}^{n}\right)\right\},
\end{array}
$$


and let $g$ be the Carathéodory function

$$
g(x, v):=f\left(x, u(x), \ldots, \nabla^{s-1} u(x), v\right) .
$$

Reasoning as in Lemma 3.4, it is easy to show that $\mathcal{F}(u ; \cdot)$ is the trace of a Radon measure absolutely continuous with respect to $\mathcal{L}^{N}\lfloor\Omega$.

For any function $v \in L^{p}\left(\Omega ; E_{s}^{n}\right)$ set

$$
\begin{gathered}
\mathcal{G}(v ; D):=\inf \left\{\liminf _{k \rightarrow \infty} \int_{D} g\left(x, V_{k}(x)\right) d x:\left\{V_{k}\right\} \subset L^{p}\left(D ; E_{s}^{n}\right) \cap \operatorname{ker} \mathcal{A} \text { is p-equi-integrable },\right. \\
\text { and } \left.\quad V_{k} \rightarrow v \text { in } L^{p}\left(D ; E_{s}^{n}\right)\right\},
\end{gathered}
$$

where the differential operator $\mathcal{A}$ is given by

$$
\mathcal{A} v:=\left(\frac{\partial}{\partial x_{i}} v_{i_{1} \ldots i_{h} j i_{h+2} \ldots i_{s}}-\frac{\partial}{\partial x_{j}} v_{i_{1} \ldots i_{h} i i_{h+2} \ldots i_{s}}\right)_{0 \leq h \leq s-1,1 \leq i, j, i_{1} \ldots i_{s} \leq N} .
$$

Here $h=0$ and $h=s-1$ correspond to the multi-indeces $j i_{2} \ldots i_{s}$ and $i_{1} \ldots i_{s-1} j$. By Theorem 3.6 (and Cor. 3.2), and where the target space $\mathbb{R}^{d}$ is being replaced by the finite dimensional vector space $E_{s}^{n}$, for any $D \in \mathcal{O}(\Omega)$

$$
\mathcal{G}(v ; D)=\int_{D} \mathcal{Q}_{\mathcal{A}} g(x, v(x)) d x,
$$

where for a.e. $x \in \Omega$ and for all $v \in E_{s}^{n}$,

$$
\mathcal{Q}_{\mathcal{A}} g(x, v):=\inf \left\{\int_{Q} g(x, v+w(y)) d y: w \in C_{1-\text { per }}^{\infty}\left(\mathbb{R}^{N} ; E_{s}^{n}\right) \cap \operatorname{ker} \mathcal{A}, \int_{Q} w(y) d y=0\right\} .
$$

As shown in [22],

$$
\left\{w \in C_{1-\text { per }}^{\infty}\left(\mathbb{R}^{N} ; E_{s}^{n}\right): \mathcal{A} w=0, \quad \int_{Q} w d x=0\right\}=\left\{\nabla^{s} \varphi: \varphi \in C_{1-\text { per }}^{\infty}\left(\mathbb{R}^{N} ; \mathbb{R}^{n}\right)\right\} .
$$

Hence

$$
\mathcal{Q}_{\mathcal{A}} g(x, v)=\inf \left\{\int_{Q} g\left(x, v+\nabla^{s} \varphi(y)\right) d y: \varphi \in C_{1-\text { per }}^{\infty}\left(\mathbb{R}^{N} ; \mathbb{R}^{N}\right)\right\}
$$

In particular

$$
\mathcal{G}\left(\nabla^{s} u ; D\right)=\int_{D} \mathcal{Q}^{s} f\left(x, u, \ldots, \nabla^{s} u\right) d x
$$

Let $\left\{u_{k}\right\} \subset W^{s, p}\left(\Omega ; \mathbb{R}^{n}\right)$ be any sequence such that $u_{k} \rightarrow u$ in $W^{s, p}\left(\Omega ; \mathbb{R}^{n}\right)$. Extracting a subsequence, if necessary, we may assume that

$$
\mathbf{u}_{k}:=\left(u_{k}, \ldots, \nabla^{s-1} u_{k}\right) \rightarrow \mathbf{u}:=\left(u, \ldots, \nabla^{s-1} u\right) \quad \text { in } L^{p}\left(D ; E_{[s-1]}^{n}\right) .
$$


Since $\nabla^{s} u_{k} \rightarrow \nabla^{s} u$ in $L^{p}\left(D ; E_{s}^{n}\right)$ and $\mathcal{A} \nabla^{s} u_{k}=0$, by Lemma 3.1 there exists a $p$-equi-integrable sequence $\left\{V_{k}\right\} \subset L^{p}\left(D ; E_{s}^{n}\right) \cap \operatorname{ker} \mathcal{A}$ such that $V_{k} \rightarrow \nabla^{s} u$ in $L^{p}\left(D ; E_{s}^{n}\right)$ and

$$
\liminf _{k \rightarrow \infty} \int_{D} g\left(x, V_{k}(x)\right) d x \leq \liminf _{k \rightarrow \infty} \int_{\Omega} f\left(x, u_{k}, \ldots, \nabla^{s} u_{k}\right) d x .
$$

Thus

$$
\mathcal{G}\left(\nabla^{s} u ; D\right) \leq \mathcal{F}(u ; D) .
$$

To prove the converse inequality, fix $x_{0} \in \Omega$ and $r>0$, and consider any $p$-equi-integrable sequence $\left\{V_{k}\right\} \subset$ $L^{p}\left(B\left(x_{0} ; r\right) ; E_{s}^{n}\right) \cap \operatorname{ker} \mathcal{A}$ such that $V_{k} \rightarrow \nabla^{s} u$ in $L^{p}\left(B\left(x_{0} ; r\right) ; E_{s}^{n}\right)$. An induction argument, similar to the one used in [22] to prove (4.1) above, shows that $\mathcal{A} V_{k}=0$ if and only if there exists $\varphi_{k} \in W^{s, p}\left(B\left(x_{0} ; r\right) ; \mathbb{R}^{n}\right)$ such that $\nabla^{s} \varphi_{k}=V_{k}$. By Lemmas 1.1-1.3 in [24], for any $\varphi \in W^{s, p}\left(B\left(x_{0} ; r\right) ; \mathbb{R}^{n}\right)$ we may find a unique function $P \in C^{\infty}\left(\mathbb{R}^{N} ; \mathbb{R}^{n}\right)$ whose components are polynomials of degree $s-1$ such that

$$
\int_{B\left(x_{0}, r\right)} \nabla^{l}(\varphi-P) d x=0 \quad 0 \leq l \leq s-1,
$$

and a constant $C(n, N, s, p, r)>0$ such that the following Poincaré type inequality holds

$$
\|\varphi-P\|_{W^{s, p}\left(B\left(x_{0} ; r\right) ; \mathbb{R}^{n}\right)} \leq C\left\|\nabla^{s} \varphi\right\|_{L^{p}\left(B\left(x_{0} ; r\right) ; E_{s}^{n}\right)} .
$$

Let $P_{k}$ and $P$ be the functions associated to $\varphi_{k}$ and $u$, respectively, and satisfying $(4.4,4.5)$. Since $\nabla^{s} \varphi_{k} \rightarrow \nabla^{s} u$ in $L^{p}\left(B\left(x_{0} ; r\right) ; E_{s}^{n}\right)$, we have that

$$
\varphi_{k}-P_{k} \rightarrow u-P \text { in } W^{s, p}\left(B\left(x_{0} ; r\right) ; \mathbb{R}^{n}\right),
$$

so

$$
u_{k}:=\varphi_{k}-P_{k}+P \rightarrow u \text { in } W^{s, p}\left(B\left(x_{0} ; r\right) ; \mathbb{R}^{N}\right) .
$$

Consider a subsequence of $\left\{V_{k}\right\}$ (not relabelled) such that the two sequences

$$
\left\{\left(u_{k}, \ldots, \nabla^{s-1} u_{k}, V_{k}\right)\right\} \quad \text { and } \quad\left\{\left(u, \ldots, \nabla^{s-1} u, V_{k}\right)\right\}
$$

generate the Young measure $\left\{\delta_{\left(u(x), \ldots, \nabla^{s-1} u\right)} \otimes \nu_{x}\right\}_{x \in B\left(x_{0}, r\right)}$, and

$$
\left(u_{k}, \ldots, \nabla^{s-1} u_{k}\right) \rightarrow\left(u, \ldots, \nabla^{s-1} u\right)
$$

pointwise and in $L^{p}\left(B\left(x_{0} ; r\right) ; E_{s-1}^{n}\right)$. Since $\left\{V_{k}\right\}$ is $p$-equi-integrable and $u_{k}$ converge to $u$ strongly in $W^{s-1, p}\left(\Omega ; \mathbb{R}^{N}\right)$, it follows from Theorem 2.1 and the growth condition on $f$ that

$$
\lim _{k \rightarrow \infty} \int_{B\left(x_{0}, r\right)} f\left(u_{k}, \ldots, \nabla^{s-1} u_{k}, V_{k}\right) d x=\lim _{k \rightarrow \infty} \int_{B\left(x_{0}, r\right)} g\left(x, V_{k}(x)\right) d x .
$$

Thus

$$
\mathcal{G}\left(\nabla^{s} u ; B\left(x_{0}, r\right)\right) \geq \mathcal{F}\left(u ; B\left(x_{0}, r\right)\right)
$$

which, together with (4.3), yields

$$
\mathcal{G}\left(\nabla^{s} u ; B\left(x_{0}, r\right)\right)=\mathcal{F}\left(u ; B\left(x_{0}, r\right)\right)
$$


Since $\mathcal{F}(u ; \cdot)$ and $\mathcal{G}\left(\nabla^{s} u ; \cdot\right)$ are both traces of a Radon measures absolutely continuous with respect to $\mathcal{L}^{N}\lfloor\Omega$, by (4.2) and (4.6) we immediately obtain that

$$
\mathcal{F}(u ; D)=\mathcal{G}\left(\nabla^{s} u ; D\right)=\int_{D} \mathcal{Q} f\left(x, u, \ldots, \nabla^{s} u\right) d x
$$

Proof of Theorem 1.5. We only proof Theorem 1.5 for $1 \leq p<\infty$, the case $p=\infty$ being very similar.

For $v \in \mathbb{R}^{N^{2}-1}$ let

$$
v=\left(v^{(1)}, \ldots, v^{(N)}\right), \quad \text { where } \quad v^{(i)} \in \mathbb{R}^{N}, \quad i=1, \ldots, N-1, \quad v^{(N)} \in \mathbb{R}^{N-1} .
$$

Given a function $v \in L^{p}\left(\Omega ; \mathbb{R}^{N^{2}-1}\right)$ define the differential operator $\mathcal{A}$ as follows

$$
\mathcal{A} v:=\left(\begin{array}{c}
\operatorname{curl} v^{(1)} \\
\vdots \\
\operatorname{curl} v^{(N-1)} \\
\operatorname{curl}\left(v^{(N)},-v_{1}^{(1)}-\ldots-v_{N-1}^{(N-1)}\right)
\end{array}\right)
$$

A straightforward calculation shows that $\mathcal{A}$ satisfies the constant-rank property (1.1).

Given a Carathéodory function $f: \Omega \times \mathbb{R}^{N} \times \mathbb{R}^{N^{2}} \rightarrow[0, \infty)$, we define $\hat{f}(x, u, v)$, for $(x, u, v) \in \Omega \times \mathbb{R}^{N} \times \mathbb{R}^{N^{2}-1}$, as

$$
\hat{f}(x, u, v)=f\left(x, u,\left(v^{(1)}, \ldots, v^{(N-1)},\left(\begin{array}{c}
v^{(N)} \\
-v_{1}^{(1)}-\ldots-v_{N-1}^{(N-1)}
\end{array}\right)\right)\right) .
$$

Let $u \in W^{1, p}\left(\Omega ; \mathbb{R}^{N}\right)$, with $\operatorname{div} u=0$, and let $\left\{u_{n}\right\} \subset W^{1, p}\left(\Omega ; \mathbb{R}^{N}\right)$ be such that $\operatorname{div} u_{n}=0$ and $u_{n} \rightarrow u$ in $W^{1, p}\left(\Omega ; \mathbb{R}^{N}\right)$. By Lemma 3.1 there exists a $p$-equi-integrable sequence $\left\{V_{n}\right\} \subset L^{p}\left(\Omega ; \mathbb{R}^{N^{2}-1}\right) \cap$ ker $\mathcal{A}$ such that $V_{n} \rightarrow v$ in $L^{p}\left(D ; \mathbb{R}^{N^{2}-1}\right)$ and

$$
\liminf _{n \rightarrow \infty} \int_{\Omega} \hat{f}\left(x, u, V_{n}\right) d x \leq \liminf _{n \rightarrow \infty} \int_{\Omega} \hat{f}\left(x, u_{n}, v_{n}\right) d x=\liminf _{n \rightarrow \infty} \int_{\Omega} f\left(x, u_{n}, \nabla u_{n}\right) d x
$$

where

$$
v_{n}:=\left(\nabla u_{n}^{(1)}, \ldots, \nabla u_{n}^{(N-1)},\left(\begin{array}{c}
\frac{\partial u_{n}^{(N)}}{\partial x_{1}} \\
\vdots \\
\frac{\partial u_{n}^{(N)}}{\partial x_{N-1}}
\end{array}\right)\right), \quad v:=\left(\nabla u^{(1)}, \ldots, \nabla u^{(N-1)},\left(\begin{array}{c}
\frac{\partial u^{(N)}}{\partial x_{1}} \\
\vdots \\
\frac{\partial u^{(N)}}{\partial x_{N-1}}
\end{array}\right)\right)
$$

Define

$$
\begin{gathered}
\mathcal{G}(v ; D):=\inf \left\{\liminf _{n \rightarrow \infty} \int_{D} \hat{g}\left(x, V_{n}(x)\right) d x:\left\{V_{n}\right\} \subset L^{p}\left(D ; \mathbb{R}^{N^{2}-1}\right) \cap \operatorname{ker} \mathcal{A} \text { is } p\right. \text {-equi-integrable } \\
\text { and } \left.V_{n} \rightarrow v \text { in } L^{p}\left(D ; \mathbb{R}^{N^{2}-1}\right)\right\}
\end{gathered}
$$


where $\hat{g}$ is the Carathéodory function defined by $\hat{g}(x, v):=\hat{f}(x, u(x), v)$. By Theorem 3.6 (and Cor. 3.2)

$$
\mathcal{G}(v ; \Omega)=\int_{\Omega} \mathcal{Q}_{\mathcal{A}} \hat{g}(x, v) d x
$$

where

$$
\mathcal{Q}_{\mathcal{A}} \hat{g}(x, v(x)):=\inf \left\{\int_{Q} \hat{f}(x, u(x), v(x)+w(y)) d y: w \in C_{1 \text {-per }}^{\infty}\left(\mathbb{R}^{N} ; \mathbb{R}^{N^{2}-1}\right) \cap \operatorname{ker} \mathcal{A}, \quad \int_{Q} w(y) d y=0\right\} .
$$

Now

$$
w \in C_{1 \text {-per }}^{\infty}\left(\mathbb{R}^{N} ; \mathbb{R}^{N^{2}-1}\right) \cap \operatorname{ker} \mathcal{A} \text { and } \int_{Q} w(y) d y=0
$$

if and only if there exists $\varphi \in C_{1-\text { per }}^{\infty}\left(\mathbb{R}^{N} ; \mathbb{R}^{N}\right)$ such that

$$
w=\left(\nabla \varphi^{(1)}, \ldots, \nabla \varphi^{(N-1)},\left(\begin{array}{c}
\frac{\partial \varphi^{(N)}}{\partial x_{1}} \\
\vdots \\
\frac{\partial \varphi^{(N)}}{\partial x_{N-1}}
\end{array}\right)\right)
$$

and $\frac{\partial \varphi^{(N)}}{\partial x_{N}}=-\frac{\partial \varphi^{(1)}}{\partial x_{1}}-\ldots-\frac{\partial \varphi^{(N-1)}}{\partial x_{N-1}}$. Hence

$$
\begin{aligned}
\mathcal{Q}_{\mathcal{A}} \hat{g}(x, v(x)) & =\inf \left\{\int_{Q} f(x, u(x), \nabla u(x)+\nabla \varphi(y)) d y: \varphi \in C_{1-\mathrm{per}}^{\infty}\left(\mathbb{R}^{N} ; \mathbb{R}^{N}\right), \quad \operatorname{div} \varphi=0\right\} \\
& =\bar{f}(x, u(x), \nabla u(x)) .
\end{aligned}
$$

Thus, by $(4.7,4.9)$, and $(4.10)$,

$$
\int_{\Omega} \bar{f}(x, u(x), \nabla u(x)) d x=\mathcal{G}(v ; \Omega) \leq \liminf _{n \rightarrow \infty} \int_{\Omega} \hat{f}\left(x, u, V_{n}\right) d x \leq \liminf _{n \rightarrow \infty} \int_{\Omega} f\left(x, u_{n}(x), \nabla u_{n}(x)\right) d x,
$$

and, in turn,

$$
\begin{gathered}
\int_{\Omega} \bar{f}(x, u(x), \nabla u(x)) d x \leq \inf \left\{\liminf _{n \rightarrow \infty} \int_{\Omega} f\left(x, u_{n}(x), \nabla u_{n}(x)\right) d x:\left\{u_{n}\right\} \subset W^{1, p}\left(\Omega ; \mathbb{R}^{N}\right),\right. \\
\left.\operatorname{div} u_{n}=0, \quad u_{n} \rightarrow u \text { in } W^{1, p}\left(\Omega ; \mathbb{R}^{N}\right)\right\} .
\end{gathered}
$$

To prove the converse inequality, fix $\varepsilon>0$. By the definition of $\mathcal{G}(v ; \Omega)$, there exists a $p$-equi-integrable sequence $\left\{V_{n}\right\} \subset L^{p}\left(D ; \mathbb{R}^{N^{2}-1}\right) \cap \operatorname{ker} \mathcal{A}$ such that $V_{n} \rightarrow v$ in $L^{p}\left(D ; \mathbb{R}^{N^{2}-1}\right)$ and

$$
\int_{\Omega} \bar{f}(x, u(x), \nabla u(x)) d x+\varepsilon>\liminf _{n \rightarrow \infty} \int_{D} \hat{g}\left(x, V_{n}(x)\right) d x=\liminf _{n \rightarrow \infty} \int_{D} \hat{f}\left(x, u(x), V_{n}(x)\right) d x,
$$

where we used for $v$ the notation introduced in (4.8). Now $\mathcal{A} V_{n}=0$ if and only if there exists $\varphi_{n} \in W^{1, p}\left(\Omega ; \mathbb{R}^{N}\right)$ such that

$$
V_{n}=\left(\nabla \varphi_{n}^{(1)}, \ldots, \nabla \varphi_{n}^{(N-1)},\left(\begin{array}{c}
\frac{\partial \varphi_{n}^{(N)}}{\partial x_{1}} \\
\vdots \\
\frac{\partial \varphi_{n}^{(N)}}{\partial x_{N-1}}
\end{array}\right)\right)
$$


and $\frac{\partial \varphi_{n}^{(N)}}{\partial x_{N}}=-\frac{\partial \varphi_{n}^{(1)}}{\partial x_{1}}-\ldots-\frac{\partial \varphi_{n}^{(N-1)}}{\partial x_{N-1}}$. Since $\nabla \varphi_{n} \rightarrow \nabla u$ in $L^{p}\left(\Omega ; \mathbb{R}^{N^{2}}\right)$, we have that

$$
\varphi_{n}-\frac{1}{|\Omega|} \int_{\Omega} \varphi_{n}(x) d x \rightarrow U \text { in } W^{1, p}\left(\Omega ; \mathbb{R}^{N}\right),
$$

where $U=u+c$ for some constant $c \in \mathbb{R}^{N}$. So

$$
u_{n}:=\varphi_{n}-\frac{1}{|\Omega|} \int_{\Omega} \varphi_{n}(x) d x-c \rightarrow u \text { in } W^{1, p}\left(\Omega ; \mathbb{R}^{N}\right),
$$

and $\operatorname{div} u_{n}=0$. Consider a subsequence $\left\{V_{n_{k}}\right\}$ of $\left\{V_{n}\right\}$ such that

$$
\lim _{k \rightarrow \infty} \int_{\Omega} \hat{f}\left(x, u(x), V_{n_{k}}(x)\right) d x=\liminf _{n \rightarrow \infty} \int_{\Omega} \hat{f}\left(x, u(x), V_{n}(x)\right) d x
$$

and $\left\{\left(u_{n_{k}}, V_{n_{k}}\right)\right\}$ and $\left\{\left(u, V_{n_{k}}\right)\right\}$ generates the Young measure $\left\{\delta_{u(x)} \otimes \nu_{x}\right\}_{x \in \Omega}$. Since $\left\{V_{n_{k}}\right\}$ is $p$-equi-integrable and $u_{n_{k}}$ converge to $u$ strongly in $L^{p}\left(\Omega ; \mathbb{R}^{N}\right)$, it follows from Theorem 2.1 and the growth condition on $f$ that

$$
\lim _{k \rightarrow \infty} \int_{\Omega} \hat{f}\left(x, u(x), V_{n_{k}}(x)\right) d x=\lim _{k \rightarrow \infty} \int_{\Omega} \hat{f}\left(x, u_{n_{k}}(x), V_{n_{k}}(x)\right) d x .
$$

By $(4.11)$

$$
\begin{array}{r}
\int_{\Omega} \bar{f}(x, u(x), \nabla u(x)) d x+\varepsilon>\lim _{k \rightarrow \infty} \int_{\Omega} \hat{f}\left(x, u_{n_{k}}(x), V_{n_{k}}(x)\right) d x=\lim _{k \rightarrow \infty} \int_{\Omega} f\left(x, u_{n_{k}}(x), \nabla u_{n_{k}}(x)\right) d x \\
\geq \inf \left\{\liminf _{n \rightarrow \infty} \int_{\Omega} f\left(x, u_{n}(x), \nabla u_{n}(x)\right) d x:\left\{u_{n}\right\} \subset W^{1, p}\left(\Omega ; \mathbb{R}^{N}\right)\right. \\
\left.\operatorname{div} u_{n}=0, \quad u_{n} \rightarrow u \text { in } W^{1, p}\left(\Omega ; \mathbb{R}^{N}\right)\right\}
\end{array}
$$

It now suffices to let $\varepsilon \rightarrow 0^{+}$.

\section{Homogenization}

In this section we will limit our analysis to the case where $1<q<\infty$.

Lemma 5.1. Let $f: \mathbb{R}^{N} \times \mathbb{R}^{d} \rightarrow[0, \infty)$ be a continuous function satisfying $\left(A_{1}\right)-\left(A_{2}\right)$. Let $v \in L^{q}\left(D ; \mathbb{R}^{d}\right) \cap$ ker $\mathcal{A}$, where $D \in \mathcal{O}(\Omega), \varepsilon_{k} \rightarrow 0^{+}$, and let $\left\{\hat{v}_{k}\right\} \subset L^{q}\left(D_{1} ; \mathbb{R}^{d}\right)$ be a sequence of functions such that

$$
\hat{v}_{k} \rightarrow v \quad \text { in } \quad L^{q}\left(D_{1} ; \mathbb{R}^{d}\right), \quad \mathcal{A} \hat{v}_{k} \rightarrow 0 \quad \text { in } \quad W^{-1, q}\left(D_{1} ; \mathbb{R}^{l}\right)
$$

for some $D_{1} \in \mathcal{O}(\Omega)$, with $D_{1} \subset D$. Then we can find a q-equi-integrable sequence $\left\{v_{k}\right\} \subset L^{q}\left(D ; \mathbb{R}^{d}\right) \cap \operatorname{ker} \mathcal{A}$ such that $\int_{D} v_{k} d x=\int_{D} v d x$,

$$
v_{k} \rightarrow v \quad \text { in } \quad L^{q}\left(D ; \mathbb{R}^{d}\right), \quad\left\|\hat{v}_{k}-v_{k}\right\|_{L^{s}\left(D_{1}\right)} \rightarrow 0 \quad \text { for all } 1 \leq s<q
$$


and

$$
\begin{aligned}
& \liminf _{k \rightarrow \infty} \int_{D_{1}} f\left(x / \varepsilon_{k}, v_{k}(x)\right) d x \leq \liminf _{k \rightarrow \infty} \int_{D_{1}} f\left(x / \varepsilon_{k}, \hat{v}_{k}(x)\right) d x, \\
& \limsup _{k \rightarrow \infty} \int_{D \backslash D_{1}}\left|v_{k}(x)\right|^{q} d x \leq \int_{D \backslash D_{1}}|v(x)|^{q} d x .
\end{aligned}
$$

Moreover, if $D=Q$, then $v_{k}=v+w_{k}$, with $w_{k} \in L_{1-\text { per }}^{q}\left(\mathbb{R}^{N} ; \mathbb{R}^{d}\right) \cap \operatorname{ker} \mathcal{A}$.

Remark 5.2. Lemma 5.1 implies, in particular, that for every $v \in L^{q}\left(\Omega ; \mathbb{R}^{d}\right) \cap \operatorname{ker} \mathcal{A}$

$$
\begin{aligned}
\Gamma-\liminf _{n \rightarrow \infty} \mathcal{F}_{\varepsilon_{n}}(v ; D)=\inf \left\{\liminf _{n \rightarrow \infty} \mathcal{F}_{\varepsilon_{n}}\left(v_{n} ; D\right): v_{n} \in L^{q}\left(D ; \mathbb{R}^{d}\right) \cap \operatorname{ker} \mathcal{A},\right. \\
\left.v_{n} \rightarrow v \operatorname{in} L^{q}\left(D ; \mathbb{R}^{d}\right), \quad \int_{D} v_{n} d x=\int_{D} v d x\right\},
\end{aligned}
$$

and if $D=Q$ then

$$
\begin{aligned}
\Gamma-\liminf _{n \rightarrow \infty} \mathcal{F}_{\varepsilon_{n}}(v ; Q)=\inf \left\{\liminf _{n \rightarrow \infty} \mathcal{F}_{\varepsilon_{n}}\left(v+w_{n} ; Q\right)\right. & : w_{n} \in L_{1-\text { per }}^{q}\left(\mathbb{R}^{N} ; \mathbb{R}^{d}\right) \cap \operatorname{ker} \mathcal{A} \\
& \left.w_{n} \rightarrow 0 \text { in } L^{q}\left(Q ; \mathbb{R}^{d}\right), \quad \int_{Q} w_{n} d x=0\right\} .
\end{aligned}
$$

Proof of Lemma 5.1. Let $g(x):=x$ in $Q$ and extend it periodically to $\mathbb{R}^{N}$ with period 1. Set $g_{k}(x):=g\left(x / \varepsilon_{k}\right)$. Since $\left\{g_{k}\right\}$ is bounded in $L^{\infty}$ and $\hat{v}_{k} \rightarrow v$ in $L^{q}\left(D_{1} ; \mathbb{R}^{d}\right)$, by Theorem 2.1 there exists a subsequence $\left\{\varepsilon_{n}\right\}$ of $\left\{\varepsilon_{k}\right\}$ such that

$$
\left\{\left(g_{n}(x), \hat{v}_{n}(x)\right)\right\} \quad \text { generates a Young measure }\left\{\nu_{x}\right\}
$$

and

For $i \in \mathbb{N}$ let

$$
\lim _{n \rightarrow \infty} \int_{D_{1}} f\left(x / \varepsilon_{n}, \hat{v}_{n}(x)\right) d x=\liminf _{k \rightarrow \infty} \int_{D_{1}} f\left(x / \varepsilon_{k}, \hat{v}_{k}(x)\right) d x .
$$

$$
F_{i}:=\left\{x \in D_{1}: \operatorname{dist}\left(x, \partial D_{1}\right)<\frac{1}{i}\right\}
$$

and consider cut-off functions $\theta_{i}$ with compact support in $D_{1}$ and such that $\theta_{i} \equiv 1$ in $D_{1} \backslash F_{i}$. Set $w_{i, n}:=$ $\theta_{i}\left(\hat{v}_{n}-v\right) \in L^{q}\left(Q ; \mathbb{R}^{d}\right)$. Then we can proceed as in the proof of Lemma 3.1 to find a $q$-equi-integrable sequence $\left\{v_{i}:=v+w_{i}\right\}$, where $\left\{w_{i}\right\}$ satisfies $(3.3,5.1)$ holds, and the two sequences

$$
\left\{\left(g_{n_{i}}(x), v_{i}(x)\right)\right\} \quad \text { and } \quad\left\{\left(g_{n_{i}}(x), \hat{v}_{n_{i}}(x)\right)\right\}
$$

generate the same Young measure $\left\{\nu_{x}\right\}$. Hence by Theorem 2.1

$$
\begin{aligned}
\lim _{i \rightarrow \infty} \int_{D_{1}} f\left(x / \varepsilon_{n_{i}}, v_{i}(x)\right) d x & =\int_{D_{1}}\left(\int_{\mathbb{R}^{N} \times \mathbb{R}^{d}} f(X, V) d \nu_{x}(X, V)\right) d x \leq \lim _{i \rightarrow \infty} \int_{D_{1}} f\left(x / \varepsilon_{n_{i}}, \hat{v}_{n_{i}}(x)\right) d x \\
& =\liminf _{k \rightarrow \infty} \int_{D_{1}} f\left(x / \varepsilon_{k}, \hat{v}_{k}(x)\right) d x,
\end{aligned}
$$

where we have used $\left(A_{2}\right)$, and the facts that $\left\{v_{i}(x)\right\}$ is $q$-equi-integrable over $D_{1}$, and that $f$ is a continuous function. 
To prove the second inequality in (5.2), we remark that by (3.3) and the fact that $\hat{w}_{i}=\theta_{i}\left(\hat{v}_{n_{i}}-v\right) \equiv 0$ outside $D_{1}$, we have for all $1 \leq s<q$

$$
\left\|v_{i}-v\right\|_{L^{s}\left(D \backslash D_{1}\right)}=\left\|\hat{w}_{i}-w_{i}\right\|_{L^{s}\left(D \backslash D_{1}\right)} \rightarrow 0 .
$$

Hence $\left\{v_{i}(x)\right\}$ generates the Young measure $\left\{\mu_{x}=\delta_{v(x)}\right\}$ on $D \backslash D_{1}$, and since $\left\{v_{i}\right\}$ is $q$-equi-integrable we have that

$$
\limsup _{i \rightarrow \infty} \int_{D \backslash D_{1}}\left|v_{i}(x)\right|^{q} d x=\int_{D \backslash D_{1}}|Y|^{q} d \mu_{x}(Y) d x=\int_{D \backslash D_{1}}|v(x)|^{q} d x .
$$

To complete the proof it suffices to define $v_{k}:=v_{n_{i}}$ for each $n_{i} \leq k<n_{i+1}$. Clearly

$$
\liminf _{k \rightarrow \infty} \int_{D_{1}} f\left(x / \varepsilon_{k}, v_{k}(x)\right) d x \leq \liminf _{i \rightarrow \infty} \int_{D_{1}} f\left(x / \varepsilon_{n_{i}}, v_{i}(x)\right) d x .
$$

Lemma 5.3. Let $\varepsilon_{n} \rightarrow 0^{+}$and let $\mathcal{R}(\Omega)$ be the family of all finite unions of open cubes contained in $\Omega$ and with vertices in $\mathbb{Q}^{N}$. Then there exists a subsequence $\left\{\varepsilon_{n_{k}}\right\}$ of $\left\{\varepsilon_{n}\right\}$ such that the $\Gamma$-limit

$$
\Gamma-\lim _{k \rightarrow \infty} \mathcal{F}_{\varepsilon_{n_{k}}}(v ; R)
$$

exists for all $v \in L^{q}\left(R ; \mathbb{R}^{d}\right) \cap \operatorname{ker} \mathcal{A}$ and for all $R \in \mathcal{R}(\Omega)$.

Proof of Lemma 5.3. Fix $R \in \mathcal{R}(\Omega)$. For simplicity set $\mathcal{F}_{n}:=\mathcal{F}_{\varepsilon_{n}}$ and let $\mathcal{B}$ denote the closed unit ball of $L^{q}\left(R ; \mathbb{R}^{d}\right)$. For each $l \in \mathbb{N}$ consider

$$
l \mathcal{B}:=\left\{v \in L^{q}\left(R ; \mathbb{R}^{d}\right):\|v\|_{L^{q}} \leq l\right\} .
$$

Since $q>1$ the dual of $L^{q}\left(R ; \mathbb{R}^{d}\right)$ is separable, and hence the space $l \mathcal{B}$ endowed with the weak topology is metrizable. Let $d_{l}$ be any metric which generates the $L^{q}$-weak topology. Consider $l=1$ and apply Proposition 2.4 to the sequence of functionals $\left\{\mathcal{F}_{n}(\cdot ; R)\right\}$ restricted to $\left(\mathcal{B} \cap \operatorname{ker} \mathcal{A}, d_{1}\right)$. Then we can find an increasing sequence of integers $\left\{n_{j}^{1}\right\}$ such that

$$
\Gamma\left(d_{1}\right)-\lim _{j \rightarrow \infty} \mathcal{F}_{n_{j}^{1}}(v ; R)
$$

exists for all $v \in \mathcal{B} \cap \operatorname{ker} \mathcal{A}$. We now proceed recursively, so that given $l \in \mathbb{N}$ we apply Proposition 2.4 to the sequence of functionals $\left\{\mathcal{F}_{n_{j}^{l-1}}(\cdot ; R)\right\}$ restricted to $\left(l \mathcal{B} \cap \operatorname{ker} \mathcal{A}, d_{l}\right)$ to obtain a subsequence $\left\{n_{j}^{l}\right\}$ of $\left\{n_{j}^{l-1}\right\}$ such that

$$
\Gamma\left(d_{l}\right)-\lim _{j \rightarrow \infty} \mathcal{F}_{n_{j}^{l}}(v ; R)
$$

exists for all $v \in l \mathcal{B} \cap \operatorname{ker} \mathcal{A}$. Let $n_{k}:=n_{k}^{k}$. Since $\left\{n_{k}\right\}$ is a subsequence of all $\left\{n_{j}^{l}\right\}$ we have that for each $l \in \mathbb{N}$

$$
\Gamma\left(d_{l}\right)-\lim _{k \rightarrow \infty} \mathcal{F}_{n_{k}}(v ; R)
$$

exists for all $v \in l \mathcal{B} \cap \operatorname{ker} \mathcal{A}$.

We claim that the $\Gamma$-limit

$$
\Gamma-\lim _{k \rightarrow \infty} \mathcal{F}_{n_{k}}(v ; R)
$$

exists for all $v \in L^{q}\left(R ; \mathbb{R}^{d}\right) \cap \operatorname{ker} \mathcal{A}$. Indeed assume by contradiction that this is not the case. Then there exists $v \in L^{q}\left(R ; \mathbb{R}^{d}\right) \cap \operatorname{ker} \mathcal{A}$ for which

$$
\mathcal{F}^{-}(v ; R):=\Gamma-\liminf _{k \rightarrow \infty} \mathcal{F}_{n_{k}}(v ; R)<\mathcal{F}^{+}(v ; R):=\Gamma-\limsup _{k \rightarrow \infty} \mathcal{F}_{n_{k}}(v ; R) .
$$


Let $v_{k} \in L^{q}\left(R ; \mathbb{R}^{d}\right) \cap \operatorname{ker} \mathcal{A}$ be such that $v_{k} \rightarrow v$ in $L^{q}\left(R ; \mathbb{R}^{d}\right)$ and

$$
\liminf _{k \rightarrow \infty} \mathcal{F}_{n_{k}}\left(v_{k} ; R\right)=\mathcal{F}^{-}(v ; R) .
$$

Since $v_{k} \rightarrow v$ in $L^{q}\left(R ; \mathbb{R}^{d}\right)$, we may find an integer $l_{0}$ such that $v_{k}, v \in l_{0} \mathcal{B} \cap \operatorname{ker} \mathcal{A}$ for all $k \in \mathbb{N}$. Consequently

$$
d_{l_{0}}\left(v_{k}, v\right) \rightarrow 0 \quad \text { as } k \rightarrow \infty,
$$

and thus

$$
\Gamma\left(d_{l_{0}}\right)-\liminf _{k \rightarrow \infty} \mathcal{F}_{n_{k}}(v ; R) \leq \liminf _{k \rightarrow \infty} \mathcal{F}_{n_{k}}\left(v_{k} ; R\right)=\mathcal{F}^{-}(v ; R)<\mathcal{F}^{+}(v ; R) \leq \Gamma\left(d_{l_{0}}\right)-\limsup _{k \rightarrow \infty} \mathcal{F}_{n_{k}}(v ; R),
$$

which contradicts the existence of the $\Gamma$-limit $\Gamma\left(d_{l_{0}}\right)-\lim _{k \rightarrow \infty} \mathcal{F}_{n_{k}}(v ; R)$, and where we have used the fact that

$$
\begin{aligned}
\mathcal{F}^{+}(v ; R) & =\inf \left\{\limsup _{k \rightarrow \infty} \mathcal{F}_{n_{k}}\left(z_{k} ; R\right): z_{k} \in L^{q}\left(R ; \mathbb{R}^{d}\right) \cap \operatorname{ker} \mathcal{A}, \quad z_{k} \rightarrow v \text { in } L^{q}\left(R ; \mathbb{R}^{d}\right)\right\} \\
& \leq \Gamma\left(d_{l_{0}}\right)-\limsup _{k \rightarrow \infty} \mathcal{F}_{n_{k}}(v ; R) \\
& =\inf \left\{\limsup _{k \rightarrow \infty} \mathcal{F}_{n_{k}}\left(z_{k} ; R\right): z_{k} \in l_{0} \mathcal{B} \cap \operatorname{ker} \mathcal{A}, \quad z_{k} \rightarrow v \text { in } L^{q}\left(R ; \mathbb{R}^{d}\right)\right\} .
\end{aligned}
$$

Hence (5.3) holds. To conclude the proof of the lemma it suffices to observe that since the family $\mathcal{R}(\Omega)$ is countable, with a diagonal process it is possible to extract a further subsequence for which (5.3) holds for all $R \in \mathcal{R}(\Omega)$.

Remark 5.4. The previous proof asserts that for any given $D \in \mathcal{O}(\Omega)$ and $\varepsilon_{n} \rightarrow 0^{+}$there exists a subsequence $\left\{\varepsilon_{n_{k}}\right\}$ (depending on the particular set $D$ ) of $\left\{\varepsilon_{n}\right\}$ such that such that the $\Gamma$-limit

$$
\Gamma-\lim _{k \rightarrow \infty} \mathcal{F}_{\varepsilon_{n_{k}}}(v ; D)
$$

exists for all $v \in L^{q}\left(D ; \mathbb{R}^{d}\right) \cap \operatorname{ker} \mathcal{A}$.

Lemma 5.5. Assume that conditions $\left(A_{1}\right)-\left(A_{2}\right)$ hold. Given $\varepsilon_{n} \rightarrow 0^{+}$, let $\left\{\varepsilon_{n_{k}}\right\}$ be as in Lemma 5.3, and for any $D \in \mathcal{O}(\Omega)$ set

$$
\mathcal{F}^{-}(\cdot ; D):=\Gamma-\liminf _{k \rightarrow \infty} \mathcal{F}_{\varepsilon_{n_{k}}}(\cdot ; D)
$$

Then $\mathcal{F}^{-}(v ; \cdot)$ is the trace of a Radon measure.

Proof of Lemma 5.5. We start by establishing inner regularity. Precisely, we claim that for any $v \in L^{q}\left(D ; \mathbb{R}^{d}\right) \cap$ $\operatorname{ker} \mathcal{A}$ and $D \in \mathcal{O}(\Omega)$

$$
\mathcal{F}^{-}(v ; D)=\sup \left\{\mathcal{F}^{-}(v ; R): R \in \mathcal{R}(\Omega), R \subset D\right\}=\lim _{R \nearrow D} \mathcal{F}^{-}(v ; R),
$$

where the limit is taken over all finite unions of cubes $R \in \mathcal{R}(\Omega)$ with $R \subset D$. For fixed $\eta>0$ there exists $\delta>0$ such that

$$
\int_{D_{0}} C\left(1+|v(x)|^{q}\right) d x<\eta
$$


for any measurable set $D_{0} \subset D$, with $\left|D_{0}\right|<\delta$, and where $C$ is the constant given in $\left(A_{2}\right)$. Let $R \in \mathcal{R}(\Omega)$, with $R \subset D$ and $|D \backslash R|<\delta$, and, in light of Lemma 5.3, consider a sequence $\left\{\hat{v}_{k}\right\} \subset L^{q}\left(R ; \mathbb{R}^{d}\right) \cap$ ker $\mathcal{A}$, with $\hat{v}_{k} \rightarrow v$ in $L^{q}\left(R ; \mathbb{R}^{d}\right)$, and such that

$$
\lim _{k \rightarrow \infty} \mathcal{F}_{\varepsilon_{n_{k}}}\left(\hat{v}_{k} ; R\right)=\mathcal{F}^{-}(v ; R) .
$$

By Lemma 5.1 there exists a $q$-equi-integrable sequence $\left\{v_{k}\right\} \subset L^{q}\left(D ; \mathbb{R}^{d}\right) \cap \operatorname{ker} \mathcal{A}$ such that

$$
v_{k} \rightarrow v \quad \text { in } \quad L^{q}\left(D ; \mathbb{R}^{d}\right), \quad \int_{D} v_{k} d x=\int_{D} v d x
$$

and

$$
\begin{aligned}
\liminf _{k \rightarrow \infty} \int_{R} f\left(x / \varepsilon_{n_{k}}, v_{k}(x)\right) d x & \leq \lim _{k \rightarrow \infty} \int_{R} f\left(x / \varepsilon_{n_{k}}, \hat{v}_{k}(x)\right) d x, \\
\limsup _{k \rightarrow \infty} \int_{D \backslash R}\left|v_{k}(x)\right|^{q} d x & \leq \int_{D \backslash R}|v(x)|^{q} d x .
\end{aligned}
$$

Hence

$$
\begin{aligned}
\mathcal{F}^{-}(v ; D) & \leq \liminf _{k \rightarrow \infty} \int_{D} f\left(x / \varepsilon_{n_{k}}, v_{k}(x)\right) d x \leq \lim _{k \rightarrow \infty} \int_{R} f\left(x / \varepsilon_{n_{k}}, \hat{v}_{k}(x)\right) d x+\limsup _{k \rightarrow \infty} \int_{D \backslash R} C\left(1+\left|v_{k}(x)\right|^{q}\right) d x \\
& \leq \mathcal{F}^{-}(v ; R)+\int_{D \backslash R} C\left(1+|v(x)|^{q}\right) d x \leq \mathcal{F}^{-}(v ; R)+\eta
\end{aligned}
$$

where we have used $\left(A_{2}\right)$ and (5.5). Consequently

$$
\mathcal{F}^{-}(v ; D) \leq \sup \left\{\mathcal{F}^{-}(v ; R): R \in \mathcal{R}(\Omega), R \subset D\right\}+\eta,
$$

and letting $\eta \rightarrow 0^{+}$we obtain one inequality in (5.4). To show the opposite inequality, note that if $\left\{v_{k}\right\} \subset$ $L^{q}\left(D ; \mathbb{R}^{d}\right) \cap \operatorname{ker} \mathcal{A}$, with $v_{k} \rightarrow v$ in $L^{q}\left(D ; \mathbb{R}^{d}\right)$, then the restriction of $v_{k}$ to $R$ belongs to $L^{q}\left(R ; \mathbb{R}^{d}\right) \cap \operatorname{ker} \mathcal{A}$, and $v_{k} \rightarrow v$ in $L^{q}\left(R ; \mathbb{R}^{d}\right)$. Therefore

$$
\mathcal{F}^{-}(v ; R) \leq \liminf _{k \rightarrow \infty} \int_{R} f\left(x / \varepsilon_{n_{k}}, v_{k}(x)\right) d x \leq \liminf _{k \rightarrow \infty} \int_{D} f\left(x / \varepsilon_{n_{k}}, v_{k}(x)\right) d x
$$

and by taking the infimum over all such sequences we get that

$$
\mathcal{F}^{-}(v ; R) \leq \mathcal{F}^{-}(v ; D)
$$

and in turn (5.4) holds.

In order to prove that $\mathcal{F}^{-}(v ; \cdot)$ is the trace of a Radon measure, as it is usual it suffices to prove subadditivity for nested sets (see [3,21]). Let $B \subset \subset C \subset \subset D$. By (5.4) for fixed $\eta>0$ we find $R \in \mathcal{R}(\Omega)$ such that $R \subset D$ and

$$
\mathcal{F}^{-}(v ; D) \leq \eta+\mathcal{F}^{-}(v ; R)
$$

Construct $R_{1}, R_{2} \in \mathcal{R}(\Omega)$ with

$$
R \subset R_{1} \cup R_{2}, \quad R_{1} \subset D \backslash \bar{B} \text { and } \quad R_{2} \subset C .
$$


By (5.6) we have

$$
\mathcal{F}^{-}(v ; D) \leq \eta+\mathcal{F}^{-}(v ; R) \leq \eta+\mathcal{F}^{-}\left(v ; R_{1} \cup R_{2}\right)
$$

By the definition of $\Gamma$-convergence and Lemma 5.1 there exist $v_{k} \in L^{q}\left(R_{1} ; \mathbb{R}^{d}\right) \cap \operatorname{ker} \mathcal{A}$ and $w_{k} \in L^{q}\left(R_{2} ; \mathbb{R}^{d}\right) \cap$ $\operatorname{ker} \mathcal{A}$, with $v_{k} \rightarrow v$ in $L^{q}\left(R_{1} ; \mathbb{R}^{d}\right)$ and $w_{k} \rightarrow v$ in $L^{q}\left(R_{2} ; \mathbb{R}^{d}\right)$, such that

$$
\mathcal{F}^{-}\left(v ; R_{1}\right)=\lim _{k \rightarrow \infty} \mathcal{F}_{\tilde{\varepsilon}_{n_{k}}}\left(v_{k} ; R_{1}\right), \quad \mathcal{F}^{-}\left(v ; R_{2}\right)=\lim _{k \rightarrow \infty} \mathcal{F}_{\tilde{\varepsilon}_{n_{k}}}\left(w_{k} ; R_{2}\right),
$$

where $\left\{\tilde{\varepsilon}_{n_{k}}\right\}$ is a subsequence of $\left\{\varepsilon_{n_{k}}\right\}$ and $\left\{v_{k}\right\},\left\{w_{k}\right\}$ are $q$-equi-integrable over $R_{1}$ and $R_{2}$, respectively. Let $\theta_{j}$ be smooth cut-off functions which are equal to 1 on $B$ and 0 on $D \backslash \bar{C}$, and such that $\left|\left\{0<\theta_{j}<1\right\}\right| \rightarrow 0$ as $j \rightarrow \infty$. Set

For $j$ fixed

$$
\hat{V}_{j, k}:=\left(1-\theta_{j}\right) v_{k}+\theta_{j} w_{k} .
$$

$$
\mathcal{A} \hat{V}_{j, k}=\left(1-\theta_{j}\right) \mathcal{A} v_{k}+\theta_{j} \mathcal{A} w_{k}-\sum_{i=1}^{N} A^{(i)} v_{k} \frac{\partial \theta_{j}}{\partial x_{i}}+\sum_{i=1}^{N} A^{(i)} w_{k} \frac{\partial \theta_{j}}{\partial x_{i}} \rightarrow 0
$$

in $W^{-1, q}\left(R_{1} \cup R_{2} ; \mathbb{R}^{l}\right)$ strong, because $\left\|v_{k}-w_{k}\right\|_{W^{-1, q}\left(B \backslash \bar{C} ; \mathbb{R}^{l}\right)} \rightarrow 0$ as $k \rightarrow \infty$. Diagonalize to get $\hat{V}_{j}:=\hat{V}_{j, k_{j}}$ such that

$$
\hat{V}_{j} \rightarrow v \quad \text { in } \quad L^{q}\left(R_{1} \cup R_{2} ; \mathbb{R}^{d}\right), \quad \mathcal{A} \hat{V}_{j} \rightarrow 0 \quad \text { in } \quad W^{-1, q}\left(R_{1} \cup R_{2} ; \mathbb{R}^{l}\right) .
$$

By Lemma 5.1 we can find $V_{j} \in L^{q}\left(R_{1} \cup R_{2} ; \mathbb{R}^{d}\right) \cap \operatorname{ker} \mathcal{A}$ such that $V_{j} \rightarrow v$ in $L^{q}\left(R_{1} \cup R_{2} ; \mathbb{R}^{d}\right)$ and

$$
\liminf _{j \rightarrow \infty} \int_{R_{1} \cup R_{2}} f\left(x / \tilde{\varepsilon}_{n_{k_{j}}}, V_{j}(x)\right) d x \leq \liminf _{j \rightarrow \infty} \int_{R_{1} \cup R_{2}} f\left(x / \tilde{\varepsilon}_{n_{k_{j}}}, \hat{V}_{j}(x)\right) d x .
$$

Consequently, by (5.7)

$$
\begin{aligned}
\mathcal{F}^{-}(v ; D) \leq & \eta+\mathcal{F}^{-}\left(R_{1} \cup R_{2} ; D\right) \leq \eta+\liminf _{j \rightarrow \infty} \int_{R_{1} \cup R_{2}} f\left(x / \tilde{\varepsilon}_{n_{k_{j}}}, V_{j}(x)\right) d x \\
\leq & \eta+\liminf _{j \rightarrow \infty} \int_{R_{1} \cup R_{2}} f\left(x / \tilde{\varepsilon}_{n_{k_{j}}}, \hat{V}_{j}(x)\right) d x \leq \eta+\limsup _{j \rightarrow \infty} \int_{R_{1}} f\left(x / \tilde{\varepsilon}_{n_{k_{j}}}, v_{k_{j}}(x)\right) d x \\
& +\limsup _{j \rightarrow \infty} \int_{R_{2}} f\left(x / \tilde{\varepsilon}_{n_{k_{j}}}, w_{k_{j}}(x)\right) d x+\limsup _{j \rightarrow \infty} \int_{\left\{0<\theta_{j}<1\right\}} C\left(1+\left|w_{k_{j}}(x)\right|^{q}+\left|v_{k_{j}}(x)\right|^{q}\right) \\
\leq & \eta+\mathcal{F}^{-}\left(v ; R_{1}\right)+\mathcal{F}^{-}\left(v ; R_{2}\right) \leq \eta+\mathcal{F}^{-}(v ; D \backslash \bar{B})+\mathcal{F}^{-}(v ; C),
\end{aligned}
$$

where we have used (5.6) and the fact that in (5.8) inferior limits are actually limits. It now suffices to let $\eta \rightarrow 0^{+}$.

Lemma 5.6. Under conditions $\left(A_{1}\right)-\left(A_{2}\right)$, for $\mathcal{L}^{N}$ a.e. $x_{0} \in \Omega$ we have

$$
\frac{d \mathcal{F}^{-}(v ; \cdot)}{d \mathcal{L}^{N}}\left(x_{0}\right)=f_{\text {hom }}\left(v\left(x_{0}\right)\right) \text {. }
$$

Proof of Lemma 5.6. We divide the proof in three steps.

Given $\varepsilon_{n} \rightarrow 0^{+}$, let $\left\{\varepsilon_{n_{k}}\right\}$ be as in Lemma 5.3. In order to simplify the notations, in the proof of this lemma we will represent $\left\{\varepsilon_{n_{k}}\right\}$ simply by $\{\varepsilon\}$.

Step 1. We claim that

$$
\mathcal{F}^{-}\left(v\left(\cdot-x_{0}\right) ; D+x_{0}\right)=\mathcal{F}^{-}(v ; D)
$$


The proof is similar to the one of Lemma 3.9 in [11]. We present it here for the convenience of the reader. Let $v_{\varepsilon} \in L^{q}\left(D ; \mathbb{R}^{d}\right) \cap \operatorname{ker} \mathcal{A}$ be such that $v_{\varepsilon} \rightarrow v$ in $L^{q}\left(D ; \mathbb{R}^{d}\right)$ and

$$
\mathcal{F}^{-}(v ; D)=\liminf _{\varepsilon \rightarrow 0} \mathcal{F}_{\varepsilon}\left(v_{\varepsilon} ; D\right)
$$

Consider the sequence $z_{\varepsilon}:=\left[x_{0} / \varepsilon\right] \in \mathbb{Z}^{N}$, so that $x_{\varepsilon}:=z_{\varepsilon} \varepsilon$ converges to $x_{0}$. Here $[z]:=\left(\left[z_{1}\right], \ldots\left[z_{N}\right]\right)$, with $\left[z_{i}\right]$ denoting the integer part of $z_{i} \in \mathbb{R}$. By the periodicity of $f$,

$$
\mathcal{F}_{\varepsilon}\left(v_{\varepsilon} ; D\right)=\int_{D} f\left(\frac{x+x_{\varepsilon}}{\varepsilon}, v_{\varepsilon}(x)\right) d x=\int_{D+x_{\varepsilon}} f\left(\frac{y}{\varepsilon}, v_{\varepsilon}\left(y-x_{\varepsilon}\right)\right) d y .
$$

Let $B \subset \subset D$. For $\varepsilon$ sufficiently small we have that $D+x_{\varepsilon} \supset B+x_{0}$, and thus

$$
\mathcal{F}_{\varepsilon}\left(v_{\varepsilon} ; D\right) \geq \int_{B+x_{0}} f\left(\frac{y}{\varepsilon}, v_{\varepsilon}\left(y-x_{\varepsilon}\right)\right) d y
$$

Since $v_{\varepsilon}\left(\cdot-x_{\varepsilon}\right) \rightarrow v\left(\cdot-x_{0}\right)$ in $L^{q}\left(B+x_{0} ; \mathbb{R}^{d}\right)$, and $v_{\varepsilon}\left(\cdot-x_{\varepsilon}\right) \in L^{q}\left(B+x_{0} ; \mathbb{R}^{d}\right) \cap$ ker $\mathcal{A}$, by $(5.9,5.10)$, we obtain

$$
\mathcal{F}^{-}(v ; D) \geq \mathcal{F}^{-}\left(v\left(\cdot-x_{0}\right) ; B+x_{0}\right)
$$

By letting $R \nearrow D+x_{0}, R \in \mathcal{R}(\Omega)$, setting $B:=R-x_{0}$ above, we obtain by (5.4)

$$
\mathcal{F}^{-}(v ; D) \geq \mathcal{F}^{-}\left(v\left(\cdot-x_{0}\right) ; D+x_{0}\right)
$$

The converse inequality follows in a similar way.

Step 2. Next, we show that

$$
\frac{d \mathcal{F}^{-}(v ; \cdot)}{d \mathcal{L}^{N}}\left(x_{0}\right) \geq f_{\text {hom }}\left(v\left(x_{0}\right)\right) \quad \text { for } \mathcal{L}^{N} \text { a.e. } x_{0} \in \Omega \text {. }
$$

Fix $x_{0} \in \Omega$ such that

$$
\lim _{r \rightarrow 0^{+}} \frac{1}{r^{N}} \int_{Q\left(x_{0}, r\right)}\left|v(x)-v\left(x_{0}\right)\right|^{q} d x=0
$$

and

$$
\frac{d \mathcal{F}^{-}(v ; \cdot)}{d \mathcal{L}^{N}}\left(x_{0}\right)=\lim _{r \rightarrow 0^{+}} \liminf _{\varepsilon \rightarrow 0^{+}} \frac{1}{r^{N}} \int_{r Q} f\left(\frac{x}{\varepsilon}, v_{\varepsilon, r}(x)\right) d x<\infty,
$$

where we have used Step 1 and Lemma 5.5, and where we have chosen the radii $r \rightarrow 0^{+}$such that $\mathcal{F}^{-}\left(v\left(\cdot+x_{0}\right) ; \partial(r Q)\right)=0$. Here $v_{\varepsilon, r} \in L^{q}\left(r Q ; \mathbb{R}^{d}\right) \cap \operatorname{ker} \mathcal{A}$ and $v_{\varepsilon, r} \rightarrow v\left(\cdot+x_{0}\right)$ in $L^{q}\left(r Q ; \mathbb{R}^{d}\right)$ as $\varepsilon \rightarrow 0^{+}$. Then

$$
\frac{d \mathcal{F}^{-}(v ; \cdot)}{d \mathcal{L}^{N}}\left(x_{0}\right)=\lim _{r \rightarrow 0^{+}} \liminf _{\varepsilon \rightarrow 0^{+}} \int_{Q} f\left(\frac{r}{\varepsilon} y, v\left(x_{0}\right)+w_{\varepsilon, r}(y)\right) d y
$$

where $w_{\varepsilon, r}(y):=v_{\varepsilon, r}(r y)-v\left(x_{0}\right)$. As in the proof of Lemma 3.5, we have that $w_{\varepsilon, r} \rightarrow 0$ in $L^{q}\left(Q ; \mathbb{R}^{d}\right)$ if we first let $\varepsilon \rightarrow 0$ and then $r \rightarrow 0^{+}$. Diagonalize to get $\hat{w}_{k} \in L^{q}\left(Q ; \mathbb{R}^{d}\right) \cap \operatorname{ker} \mathcal{A}$ such that $\hat{w}_{k} \rightarrow 0$ in $L^{q}\left(Q ; \mathbb{R}^{d}\right)$,

$$
\frac{d \mathcal{F}^{-}(v ; \cdot)}{d \mathcal{L}^{N}}\left(x_{0}\right)=\lim _{k \rightarrow \infty} \int_{Q} f\left(s_{k} y, v\left(x_{0}\right)+\hat{w}_{k}(y)\right) d y
$$


and where $s_{k}:=1 / \varepsilon_{k} \rightarrow \infty$. By Lemma 5.1, applied to the Carathéodory function $h(x, v):=f\left(x, v\left(x_{0}\right)+v\right)$, there exists a $q$-equi-integrable sequence $\left\{w_{k}\right\} \subset L_{1 \text {-per }}^{q}\left(\mathbb{R}^{N} ; \mathbb{R}^{d}\right) \cap \operatorname{ker} \mathcal{A}$ such that

$$
w_{k} \rightarrow 0 \quad \text { in } \quad L^{q}\left(Q ; \mathbb{R}^{d}\right), \quad \int_{Q} w_{k} d y=0
$$

and

Consequently

$$
\liminf _{k \rightarrow \infty} \int_{Q} f\left(s_{k} y, v\left(x_{0}\right)+w_{k}(y)\right) d y \leq \lim _{k \rightarrow \infty} \int_{Q} f\left(s_{k} y, v\left(x_{0}\right)+\hat{w}_{k}(y)\right) d y
$$

$$
\frac{d \mathcal{F}^{-}(v ; \cdot)}{d \mathcal{L}^{N}}\left(x_{0}\right) \geq \liminf _{k \rightarrow \infty} \int_{Q} f\left(s_{k} y, v\left(x_{0}\right)+w_{k}(y)\right) d y \geq \liminf _{i \rightarrow \infty} \liminf _{k \rightarrow \infty} \int_{Q} f\left(s_{k} y, v\left(x_{0}\right)+\theta_{i}(y) w_{k}(y)\right) d y,
$$

where $0 \leq \theta_{i} \leq 1$ are smooth cut-off functions with compact support in $Q$ such that $\theta_{i} \equiv 1$ in $(1-1 / i) Q$, and where we used the $q$-equi-integrability of $\left\{w_{k}\right\}$ and $\left(A_{2}\right)$. Then $\theta_{i} w_{k} \rightarrow 0$ in $L^{q}\left(Q ; \mathbb{R}^{d}\right)$ as $k \rightarrow \infty$ and $i \rightarrow \infty$, in this order, and

$$
\lim _{i \rightarrow \infty} \lim _{k \rightarrow \infty} \mathcal{A}\left(\theta_{i} w_{k}\right)=0 \quad \text { in } \quad W^{-1, q}\left(Q ; \mathbb{R}^{l}\right) .
$$

Diagonalize to get $U_{i}:=\theta_{i} w_{k_{i}}$ extended by zero outside $Q$, such that $U_{i} \rightarrow 0$ in $L^{q}\left(Q ; \mathbb{R}^{d}\right), \mathcal{A} U_{i} \rightarrow 0$ in $W^{-1, q}\left(Q ; \mathbb{R}^{l}\right)$ as $i \rightarrow \infty$, and

$$
\liminf _{i \rightarrow \infty} \liminf _{k \rightarrow \infty} \int_{Q} f\left(s_{k} y, v\left(x_{0}\right)+\theta_{i}(y) w_{k}(y)\right) d y=\liminf _{i \rightarrow \infty} \int_{Q} f\left(s_{k_{i}}, v\left(x_{0}\right)+U_{i}(y)\right) d y .
$$

Thus

$$
\begin{aligned}
\frac{d \mathcal{F}^{-}(v ; \cdot)}{d \mathcal{L}^{N}}\left(x_{0}\right) & \geq \liminf _{i \rightarrow \infty} \frac{1}{s_{k_{i}}^{N}} \int_{s_{k_{i}} Q} f\left(x, v\left(x_{0}\right)+U_{i}\left(\frac{x}{s_{k_{i}}}\right)\right) d x \\
& \geq \liminf _{i \rightarrow \infty} \frac{1}{s_{k_{i}}^{N}} \int_{\left(\left[s_{k_{i}}\right]+1\right) Q} f\left(x, v\left(x_{0}\right)+U_{i}\left(\frac{x}{s_{k_{i}}}\right)\right) d x \\
& -\limsup _{i \rightarrow \infty} \frac{1}{s_{k_{i}}^{N}} \int_{\left(\left[s_{k_{i}}\right]+1\right) Q \backslash s_{k_{i}} Q} f\left(x, v\left(x_{0}\right)+U_{i}\left(\frac{x}{s_{k_{i}}}\right)\right) d x
\end{aligned}
$$

where $\left[s_{k_{i}}\right]$ denotes the integer part of $s_{k_{i}}$. We claim that the last limit is zero. Indeed

$$
\frac{1}{s_{k_{i}}^{N}} \int_{\left(\left[s_{k_{i}}\right]+1\right) Q \backslash s_{k_{i}} Q} f\left(x, v\left(x_{0}\right)+U_{i}\left(\frac{x}{s_{k_{i}}}\right)\right) d x=\int_{\frac{\left.\left(s_{k_{i}}\right]+1\right)}{s_{k_{i}}} Q \backslash Q} f\left(s_{k_{i}} y, v\left(x_{0}\right)+U_{i}(y)\right) d y .
$$

Since $\left(\left[s_{k_{i}}\right]+1\right) / s_{k_{i}} \rightarrow 1$, we have that

$$
\left|\frac{\left(\left[s_{k_{i}}\right]+1\right)}{s_{k_{i}}} Q \backslash Q\right|=\left(\frac{\left(\left[s_{k_{i}}\right]+1\right)}{s_{k_{i}}}\right)^{N}-1 \rightarrow 0,
$$

and thus the claim follows from the $q$-equi-integrability of $\left\{U_{i}\right\}$ and $\left(A_{2}\right)$. Hence, setting

$$
m_{i}:=1 / s_{k_{i}}, \quad n_{i}:=\left[s_{k_{i}}\right]+1 \in \mathbb{N},
$$

we obtain

$$
\frac{d \mathcal{F}^{-}(v ; \cdot)}{d \mathcal{L}^{N}}\left(x_{0}\right) \geq \liminf _{i \rightarrow \infty} \frac{1}{n_{i}^{N}} \int_{n_{i} Q} f\left(x, v\left(x_{0}\right)+U_{i}\left(m_{i} x\right)\right) d x=\liminf _{i \rightarrow \infty} \int_{Q} f\left(n_{i} y, v\left(x_{0}\right)+U_{i}\left(n_{i} m_{i} y\right)\right) d y .
$$


We claim that

$$
U_{i}\left(n_{i} m_{i} \cdot\right) \rightarrow 0 \quad \text { in } L^{q}\left(Q ; \mathbb{R}^{d}\right), \quad \mathcal{A} U_{i}\left(n_{i} m_{i} \cdot\right) \rightarrow 0 \quad \text { in } W^{-1, q}\left(Q ; \mathbb{R}^{l}\right)
$$

as $i \rightarrow \infty$. Assuming that the claim holds, by Lemma 5.1 there exists a $q$-equi-integrable sequence $\left\{V_{i}\right\} \subset$ $L_{1 \text {-per }}^{q}\left(\mathbb{R}^{N} ; \mathbb{R}^{d}\right) \cap \operatorname{ker} \mathcal{A}$ such that

$$
V_{i} \rightarrow 0 \quad \text { in } \quad L^{q}\left(Q ; \mathbb{R}^{d}\right), \quad \int_{Q} V_{i} d y=0
$$

and

$$
\begin{aligned}
\frac{d \mathcal{F}^{-}(v ; \cdot)}{d \mathcal{L}^{N}}\left(x_{0}\right) & \geq \liminf _{i \rightarrow \infty} \int_{Q} f\left(n_{i} y, v\left(x_{0}\right)+U_{i}\left(n_{i} m_{i} y\right)\right) d y \geq \liminf _{i \rightarrow \infty} \int_{Q} f\left(n_{i} y, v\left(x_{0}\right)+V_{i}(y)\right) d y \\
& =\liminf _{i \rightarrow \infty} \frac{1}{n_{i}^{N}} \int_{n_{i} Q} f\left(x, v\left(x_{0}\right)+V_{i}\left(\frac{x}{n_{i}^{N}}\right)\right) d x \geq f_{\text {hom }}\left(v\left(x_{0}\right)\right),
\end{aligned}
$$

and where we have used the facts that

$$
V_{i}\left(\frac{1}{n_{i}^{N}} \cdot\right) \in L_{n_{i} \text {-per }}^{q}\left(\mathbb{R}^{N} ; \mathbb{R}^{d}\right) \cap \operatorname{ker} \mathcal{A}, \quad \int_{n_{i} Q} V_{i}\left(\frac{1}{n_{i}^{N}} y\right) d y=0
$$

Thus it remains to show (5.12). If $\varphi \in C_{c}^{\infty}(Q)$ then

$$
\begin{aligned}
\int_{Q} U_{i}\left(n_{i} m_{i} y\right) \varphi(y) d y & =\frac{1}{\left(n_{i} m_{i}\right)^{N}} \int_{n_{i} m_{i} Q} U_{i}(x) \varphi\left(\frac{x}{n_{i} m_{i}}\right) d x \\
& =\frac{1}{\left(n_{i} m_{i}\right)^{N}}\left(\int_{Q} U_{i}(x) \varphi(x) d x+\int_{Q} U_{i}(x)\left(\varphi\left(\frac{x}{n_{i} m_{i}}\right)-\varphi(x)\right) d x\right),
\end{aligned}
$$

where we have used the fact that $U_{i}(x) \equiv 0$ in $n_{i} m_{i} Q \backslash Q$. Since $U_{i} \rightarrow 0$ in $L^{q}\left(Q ; \mathbb{R}^{d}\right)$ and $n_{i} m_{i} \rightarrow 1$ the first integral on the right hand side of the previous inequality tends to zero as $i \rightarrow \infty$. By Hölder's inequality

$$
\left|\int_{Q} U_{i}(x)\left(\varphi\left(\frac{x}{n_{i} m_{i}}\right)-\varphi(x)\right) d x\right| \leq\left(\sup _{l}\left\|U_{l}\right\|_{L^{q}(Q)}\right)\left(\int_{Q}\left|\varphi\left(\frac{x}{n_{i} m_{i}}\right)-\varphi(x)\right|^{q^{\prime}} d x\right)^{1 / q^{\prime}} .
$$

Since $\varphi$ is bounded we can apply Lebesgue Dominated Convergence Theorem to conclude that the right hand side approaches zero as $i \rightarrow \infty$. In a similar way we can show that

$$
\mathcal{A} U_{i}\left(n_{i} m_{i} \cdot\right) \rightarrow 0 \quad \text { in } W^{-1, q}\left(Q ; \mathbb{R}^{l}\right) \text { as } i \rightarrow \infty
$$

We omit the details.

Step 3. To conclude the proof of the lemma it remains to show that

$$
\frac{d \mathcal{F}^{-}(v ; \cdot)}{d \mathcal{L}^{N}}\left(x_{0}\right) \leq f_{\text {hom }}\left(v\left(x_{0}\right)\right) \text { for } \mathcal{L}^{N} \text { a.e. } x_{0} \in \Omega
$$

By Remark 1.8(i), for any fixed $\eta>0$ we may find $k \in \mathbb{N}, w \in L_{k \text {-per }}^{\infty}\left(\mathbb{R}^{N} ; \mathbb{R}^{d}\right) \cap \operatorname{ker} \mathcal{A}$ such that $\int_{k Q} w d x=0$ and

$$
\frac{1}{k^{N}} \int_{k Q} f\left(x, v\left(x_{0}\right)+w(x)\right) d x \leq f_{\text {hom }}\left(v\left(x_{0}\right)\right)+\eta
$$


For any fixed $r>0$ and for any $n \in \mathbb{N}$, let $u_{n, r}(x):=w(x n k / r)$. Then $u_{n, r} \in L^{\infty}\left(r Q ; \mathbb{R}^{d}\right) \cap \operatorname{ker} A, u_{n, r} \rightarrow 0$ in $L^{q}\left(r Q ; \mathbb{R}^{d}\right)$ as $n \rightarrow \infty$, and by Step 1

$$
\begin{aligned}
\frac{d \mathcal{F}^{-}(v ; \cdot)}{d \mathcal{L}^{N}}\left(x_{0}\right) & =\lim _{r \rightarrow 0^{+}} \frac{\mathcal{F}^{-}\left(v\left(\cdot+x_{0}\right) ; r Q\right)}{r^{N}} \leq \liminf _{r \rightarrow 0^{+}} \liminf _{n \rightarrow \infty} \frac{1}{r^{N}} \int_{r Q} f\left(\frac{x}{\varepsilon_{n, r}}, v\left(x_{0}+x\right)+u_{\varepsilon}(x)\right) d x \\
& =\liminf _{r \rightarrow 0^{+}} \liminf _{n \rightarrow \infty} \int_{Q} f\left(n k y, v\left(x_{0}+r y\right)+w(n k y)\right) d y
\end{aligned}
$$

where $\varepsilon_{n, r}:=r / n k$. Since $f(\cdot, v)$ is $Q$-periodic, there exists $\delta>0$ such that if $\left|v-v\left(x_{0}\right)\right|<\delta$ then

$$
\sup _{s \in \mathbb{R}^{N}}\left|f(s, v+w(s))-f\left(s, v\left(x_{0}\right)+w(s)\right)\right|<\eta .
$$

Setting $E_{r, \delta}:=\left\{y \in Q:\left|v\left(x_{0}+r y\right)-v\left(x_{0}\right)\right| \geq \delta\right\}$, we deduce that

$$
\begin{aligned}
\frac{d \mathcal{F}^{-}(v ; \cdot)}{d \mathcal{L}^{N}}\left(x_{0}\right) \leq & \eta+\limsup _{r \rightarrow 0^{+}} \limsup _{n \rightarrow \infty} \int_{Q} f\left(n k y, v\left(x_{0}\right)+w(n k y)\right) d y \\
& +\limsup _{r \rightarrow 0^{+}} \limsup _{n \rightarrow \infty} \int_{E_{r, \delta}} C\left(1+\left|v\left(x_{0}+r y\right)\right|^{q}+|w(n k y)|^{q}\right) d y \\
= & \eta+\frac{1}{k^{N}} \int_{k Q} f\left(y, v\left(x_{0}\right)+w(y)\right) d y \leq f_{\text {hom }}\left(v\left(x_{0}\right)\right)+2 \eta,
\end{aligned}
$$

where we have used (5.13), the $k Q$-periodicity of the function $h(y):=f\left(y, v\left(x_{0}\right)+w(y)\right)$, the equi-integrability of $\left\{\left|u_{\varepsilon}\right|^{q}\right\}$, and the fact that (5.11) entails

$$
\left.\lim _{r \rightarrow 0^{+}} \mid\left\{y \in Q:\left|v\left(x_{0}+r y\right)-v\left(x_{0}\right)\right| \geq \delta\right\}\right) \mid=0 .
$$

It suffices to let $\eta \rightarrow 0^{+}$.

Proof of Theorem 1.7. We claim that for any $\varepsilon_{n} \rightarrow 0^{+}$

$$
\mathcal{F}_{\text {hom }}(\cdot ; D)=\Gamma-\liminf _{n \rightarrow \infty} \mathcal{F}_{\varepsilon_{n}}(\cdot ; D) .
$$

By Lemmas 5.5 and 5.6 we always have

$$
\mathcal{F}_{\text {hom }}(\cdot ; D) \geq \Gamma-\liminf _{k \rightarrow \infty} \mathcal{F}_{\varepsilon_{n_{k}}} \geq \Gamma-\liminf _{n \rightarrow \infty} \mathcal{F}_{\varepsilon_{n}}(\cdot ; D) .
$$

Thus assume for contradiction that that there exists $\varepsilon_{n} \rightarrow 0^{+}$and $v \in L^{q}\left(D ; \mathbb{R}^{d}\right) \cap \operatorname{ker} \mathcal{A}$ such that

$$
\mathcal{F}_{\text {hom }}(v ; D)>\Gamma-\liminf _{n \rightarrow \infty} \mathcal{F}_{\varepsilon_{n}}(v ; D) .
$$

Let $\left\{v_{n}\right\} \subset L^{q}\left(D ; \mathbb{R}^{d}\right) \cap \operatorname{ker} \mathcal{A}$ be such that $v_{n} \rightarrow v$ in $L^{q}\left(D ; \mathbb{R}^{d}\right)$ and

$$
\mathcal{F}_{\text {hom }}(v ; D)>\liminf _{n \rightarrow \infty} \mathcal{F}_{\varepsilon_{n}}\left(v_{n} ; D\right),
$$

and choose a subsequence $\left\{\varepsilon_{n_{k}}\right\}$ such that

$$
\mathcal{F}_{\text {hom }}(v ; D)>\lim _{k \rightarrow \infty} \mathcal{F}_{\varepsilon_{n_{k}}}\left(v_{n_{k}} ; D\right) .
$$


Then, by the previous lemmas, we can extract a further subsequence $\left\{\varepsilon_{n_{k_{j}}}\right\}$ such that

$$
\mathcal{F}_{\text {hom }}(v ; D)=\Gamma-\liminf _{j \rightarrow \infty} \mathcal{F}_{\varepsilon_{n_{k_{j}}}}(v ; D) \leq \lim _{j \rightarrow \infty} \mathcal{F}_{\varepsilon_{n_{k_{j}}}}\left(v_{n_{k_{j}}} ; D\right)<\mathcal{F}_{\text {hom }}(v ; D),
$$

which is a contradiction and proves the claim.

Hence it remains to show that, when $\left(A_{3}\right)$ holds, for any $\varepsilon_{n} \rightarrow 0^{+}$and $v \in L^{q}\left(D ; \mathbb{R}^{d}\right) \cap \operatorname{ker} \mathcal{A}$

$$
\Gamma-\limsup _{n \rightarrow \infty} \mathcal{F}_{\varepsilon_{n}}(v ; D) \leq \mathcal{F}_{\text {hom }}(v ; D) .
$$

By taking $w_{n} \equiv v$ and using $\left(A_{2}\right)$ we get

$$
\Gamma-\limsup _{n \rightarrow \infty} \mathcal{F}_{\varepsilon_{n}}(v ; D) \leq \limsup _{n \rightarrow \infty} \mathcal{F}_{\varepsilon_{n}}\left(w_{n} ; D\right) \leq C \int_{D}\left(|v(x)|^{q}+1\right) d x .
$$

Hence for any sequence $\left\{v_{n}\right\} \subset L^{q}\left(D ; \mathbb{R}^{d}\right) \cap \operatorname{ker} \mathcal{A}$ such that $v_{n} \rightarrow v$ in $L^{q}\left(D ; \mathbb{R}^{d}\right)$ and

$$
\limsup _{n \rightarrow \infty} \mathcal{F}_{\varepsilon_{n}}\left(v_{n} ; D\right) \leq C \int_{D}\left(|v(x)|^{q}+1\right) d x,
$$

by $\left(A_{3}\right)$ we get that $\sup _{n}\left\|v_{n}\right\|_{L^{q}(D)} \leq L<\infty$, where the constant $L$ depends only on the constants in $\left(A_{2}\right),\left(A_{3}\right)$, and on $\|v\|_{L^{q}\left(\Omega ; \mathbb{R}^{d}\right)}$. Using the notation introduced in the proof of Lemma 5.3, we conclude that

$$
\Gamma-\limsup _{n \rightarrow \infty} \mathcal{F}_{\varepsilon_{n}}(v ; D)=\Gamma\left(d_{L}\right)-\limsup _{n \rightarrow \infty} \mathcal{F}_{\varepsilon_{n}}(v ; D) .
$$

By Remark 5.4 and by Lemma 5.6, for any subsequence $\left\{\varepsilon_{n_{k}}\right\}$ of $\left\{\varepsilon_{n}\right\}$ there exists a subsequence $\left\{\varepsilon_{n_{k_{j}}}\right\}$ (depending on $D$ ) such that

By (5.14) this implies that

$$
\Gamma-\lim _{j \rightarrow \infty} \mathcal{F}_{\varepsilon_{n_{k_{j}}}}(v ; D)=\mathcal{F}_{\text {hom }}(v ; D) .
$$

$$
\Gamma\left(d_{L}\right)-\lim _{j \rightarrow \infty} \mathcal{F}_{\varepsilon_{n_{k_{j}}}}(v ; D)=\mathcal{F}_{\text {hom }}(v ; D) .
$$

We can now apply the second part of Proposition 2.4 in the metric space $\left(L \mathcal{B}, d_{L}\right)$ to conclude that

$$
\Gamma\left(d_{L}\right)-\lim _{n \rightarrow \infty} \mathcal{F}_{\varepsilon_{n}}(v ; D)=\mathcal{F}_{\text {hom }}(v ; D) .
$$

Corollary 5.7. Under hypotheses $\left(A_{1}\right)-\left(A_{3}\right)$ the function $f_{\text {hom }}$ is $\mathcal{A}$-quasiconvex and the following asymptotic formula holds

$$
f_{\text {hom }}(\xi)=\lim _{T \rightarrow+\infty} \frac{1}{T^{N}} \inf \left\{\int_{T Q} f(x, \xi+v(x)) d x: v \in L_{T-\text { per }}^{q}\left(\mathbb{R}^{N} ; \mathbb{R}^{d}\right) \cap \operatorname{ker} \mathcal{A}, \int_{T Q} v(x) d x=0\right\} .
$$

Proof of Corollary 5.7. It may be shown easily, via a diagonalization procedure and in view of the coercivity condition $\left(A_{3}\right)$, that $\mathcal{F}_{\text {hom }}(\cdot ; Q)$ is $L^{q}$-sequentially weakly lower semicontinuous in $\operatorname{ker} \mathcal{A}$. In particular, this entails $\mathcal{A}$-quasiconvexity for $f_{\text {hom }}$. Indeed, fix $v \in \mathbb{R}^{d}$ and $w \in L_{1 \text {-per }}^{q}\left(\mathbb{R}^{N} ; \mathbb{R}^{d}\right) \cap \operatorname{ker} \mathcal{A}$, with $\int_{Q} w(y) d y=0$, and define $w_{n}(x):=w(n x)$. Then $w_{n} \in L^{q}\left(Q ; \mathbb{R}^{d}\right) \cap \operatorname{ker} \mathcal{A}, w_{n} \rightarrow 0$ in $L^{q}\left(Q ; \mathbb{R}^{d}\right)$, and so

$$
f_{\text {hom }}(v)=\mathcal{F}_{\text {hom }}(v ; Q) \leq \liminf _{n \rightarrow \infty} \mathcal{F}_{\text {hom }}\left(v+w_{n} ; Q\right)=\liminf _{n \rightarrow \infty} \int_{Q} f_{\text {hom }}(v+w(n x)) d x=\int_{Q} f_{\text {hom }}(v+w(x)) d x .
$$


Finally, using Theorem $1.7\left(A_{3}\right)$, and recalling Remark 5.2, we conclude that

$$
\begin{aligned}
f_{\text {hom }}(v) & =\min \left\{\mathcal{F}_{\text {hom }}(v+w ; Q): w \in L_{1-\text { per }}^{q}\left(\mathbb{R}^{N} ; \mathbb{R}^{d}\right) \cap \operatorname{ker} \mathcal{A}, \int_{Q} w(x) d x=0\right\} \\
& =\lim _{\varepsilon \rightarrow 0} \inf \left\{\mathcal{F}_{\varepsilon}(v+w ; Q): w \in L_{1-\text { per }}^{q}\left(\mathbb{R}^{N} ; \mathbb{R}^{d}\right) \cap \operatorname{ker} \mathcal{A}, \quad \int_{Q} w(x) d x=0\right\},
\end{aligned}
$$

and (5.15) follows by setting $T=1 / \varepsilon$ and changing variables in the last expression.

As a corollary of Theorem 1.7, we obtain the following result via the same choice of the underlying operator $\mathcal{A}$ as in the proof of Theorem 1.5.

Theorem 5.8 (Homogenization with constraint on the divergence). Assume that conditions $\left(A_{1}\right)-\left(A_{3}\right)$ hold, with $d=N^{2}$, and let $F_{\varepsilon}$ be defined by

$$
F_{\varepsilon}(u ; D):=\int_{D} f\left(\frac{x}{\varepsilon}, \nabla u\right) d x
$$

on functions $u \in W^{1, q}\left(\Omega ; \mathbb{R}^{N}\right)$ such that $\operatorname{div} u=0$. Then the $\Gamma$-limit

$$
F(u ; D):=\Gamma\left(L^{q}\right)-\lim _{\varepsilon \rightarrow 0} F_{\varepsilon}(u ; D)=\int_{D} f_{\mathrm{hom}}(\nabla u) d x
$$

exists on functions $u \in W^{1, q}\left(\Omega ; \mathbb{R}^{N}\right)$ such that $\operatorname{div} u=0$, where

$$
f_{\text {hom }}(v)=\inf _{k \in \mathbb{N}} \frac{1}{k^{N}} \inf \left\{\int_{k Q} f(x, v+\nabla w(x)) d x: \quad w \in W_{k \text {-per }}^{1, q}\left(\mathbb{R}^{N} ; \mathbb{R}^{N}\right), \operatorname{div} w=0\right\}
$$

for all $v \in \mathbb{R}^{N^{2}}$

\section{APPENDIX}

We prove that in Remark 1.2(iii) in the introduction

$$
\mathcal{F}(v ;(a, b))=\mathcal{F}\left(\left(v_{1}, v_{2}\right) ;(a, b)\right)=\int_{a}^{b}\left(\psi^{* *}\left(v_{1}\right)+v_{2}^{2}\right) d x
$$

where $\psi^{* *}\left(v_{1}\right)$ is the convex envelope of

$$
\psi\left(v_{1}\right):=\min \left\{\left(v_{1}-1\right)^{2},\left(v_{1}+1\right)^{2}\right\}=\left\{\begin{array}{lll}
\left(v_{1}+1\right)^{2} & \text { if } & v_{1} \geq 0 \\
\left(v_{1}-1\right)^{2} & \text { if } & v_{1}<0 .
\end{array}\right.
$$

Indeed, if $v_{1}^{n} \rightarrow v_{1}$ in $L^{2}(a, b), v_{2}^{n} \rightarrow v_{2}$ in $L^{2}(a, b)$ and $\left(v_{2}^{n}\right)^{\prime} \rightarrow 0$ in $H^{-1}(a, b)$ then the function $v_{2}$ is constant and Jensen's inequality yields

$$
\begin{aligned}
\liminf _{n \rightarrow \infty} \int_{a}^{b} f\left(v_{1}^{n}, v_{2}^{n}\right) d x & \geq \liminf _{n \rightarrow \infty} \int_{a}^{b}\left[\psi\left(v_{1}^{n}\right)+\left(v_{2}^{n}\right)^{2}\right] d x \geq \liminf _{n \rightarrow \infty} \int_{a}^{b} \psi\left(v_{1}^{n}\right) d x+\liminf _{n \rightarrow \infty} \int_{a}^{b}\left(v_{2}^{n}\right)^{2} d x \\
& \geq \int_{a}^{b} \psi^{* *}\left(v_{1}\right) d x+\left(v_{2}\right)^{2}(b-a) .
\end{aligned}
$$


The arbitrariness of the sequence $\left\{\left(v_{1}^{n}, v_{2}^{n}\right)\right\}$ allows us to conclude that

$$
\mathcal{F}(v ;(a, b)) \geq \int_{a}^{b} \psi^{* *}\left(v_{1}\right) d x+\left(v_{2}\right)^{2}(b-a) .
$$

Conversely, suppose that if $v_{1}$ is smooth, $v_{1} \in L^{2}(a, b)$, and $\left|\left\{x \in(a, b): v_{1}(x)=0\right\}\right|=0, v_{2} \in \mathbb{R}$, then

$$
\mathcal{F}(v ;(a, b)) \leq \int_{a}^{b} \psi\left(v_{1}\right) d x+\left(v_{2}\right)^{2}(b-a) .
$$

Then this inequality remains true for $v_{1} \in L^{2}(a, b), v_{2} \in \mathbb{R}$ arbitrary, because we may approximate $v_{1}$ in $L^{2}$ strong by a sequence $\left\{v_{1}^{n}\right\} \subset L^{2}(a, b) \cap C^{\infty}(a, b),\left|\left\{x \in(a, b): v_{1}^{n}(x)=0\right\}\right|=0$, and

$$
v_{1} \mapsto \mathcal{F}\left(\left(v_{1}, v_{2}\right) ;(a, b)\right), \quad v_{1} \mapsto \int_{a}^{b} \psi\left(v_{1}\right) d x
$$

are, respectively, $L^{2}$-weak lower semicontinuous and $L^{2}$-strong continuous. Once we establish $(5.17)$ for $\left(v_{1}, v_{2}\right) \in$ $L^{2}(a, b) \times \mathbb{R}$ then (5.16) follows because, once again, $\mathcal{F}\left(\left(\cdot, v_{2}\right) ;(a, b)\right)$ is $L^{2}$-weak lower semicontinuous.

Fix now $v_{1} \in L^{2}(a, b) \cap C^{\infty}(a, b)$, with $\left|\left\{x \in(a, b): v_{1}(x)=0\right\}\right|=0$, and let $v_{2} \in \mathbb{R}, \delta>0$ be fixed. Set

$$
\begin{aligned}
& A_{\delta}^{-}:=\left\{x \in(a, b):\left(v_{1}(x)-1\right)^{2}<\left(v_{1}(x)+1\right)^{2}-\delta\right\} \\
& A_{\delta}^{+}:=\left\{x \in(a, b):\left(v_{1}(x)-1\right)^{2}>\left(v_{1}(x)+1\right)^{2}+\delta\right\}
\end{aligned}
$$

If for all $\delta>0 A_{\delta}^{-}=\emptyset$ then $\left(v_{1}(x)-1\right)^{2} \geq\left(v_{1}(x)+1\right)^{2}$ for every $x \in(a, b)$ and we choose $w_{n} \notin \mathbb{Q}$ with $w_{n} \rightarrow v_{2}$. Then

$$
\begin{aligned}
\mathcal{F}\left(\left(v_{1}, v_{2}\right) ;(a, b)\right) & \leq \liminf _{n \rightarrow \infty} \int_{a}^{b} f\left(v, w_{n}\right) d x=\liminf _{n \rightarrow \infty} \int_{a}^{b}\left[\left(v_{1}(x)+1\right)^{2}+\left(w_{n}\right)^{2}\right] d x \\
& \leq \int_{a}^{b} \psi\left(v_{1}\right) d x+\left(v_{2}\right)^{2}(b-a) .
\end{aligned}
$$

Similary (5.17) holds if for all $\delta>0 A_{\delta}^{+}=\emptyset$. Thus assume that for $\delta>0$ sufficiently small $A_{\delta}^{-} \neq \emptyset \neq A_{\delta}^{+}$. Choose a cut-off function $\varphi_{\delta} \in C^{\infty}((a, b) ;[0,1])$ such that $\varphi_{\delta} \equiv 1$ in $A_{\delta}^{-}, \varphi_{\delta} \equiv 0$ in $A_{\delta}^{+}$, and let $z_{n} \in \mathbb{Q}, w_{n} \notin \mathbb{Q}$, be such that with $w_{n}, z_{n} \rightarrow v_{2}$. Define

$$
v_{2}^{n, \delta}(x):=\varphi_{\delta}(x) z_{n}+\left(1-\varphi_{\delta}(x)\right) w_{n}
$$

Since $\left(v_{2}^{n, \delta}\right)^{\prime}=\varphi_{\delta}^{\prime}(x)\left(z_{n}-w_{n}\right)$, it is clear that

$$
\lim _{\delta \rightarrow 0^{+}} \lim _{n \rightarrow \infty}\left\|v_{2}^{n, \delta}-v_{2}\right\|_{L^{2}}=\lim _{\delta \rightarrow 0^{+}} \lim _{n \rightarrow \infty}\left\|\left(v_{2}^{n, \delta}\right)^{\prime}\right\|_{H^{-1}}=0
$$


We have

$$
\begin{aligned}
\mathcal{F}\left(\left(v_{1}, v_{2}\right) ;(a, b)\right) & \leq \liminf _{\delta \rightarrow 0^{+}} \liminf _{n \rightarrow \infty} \int_{a}^{b} f\left(v_{1}, v_{2}^{n, \delta}\right) d x \\
& =\liminf _{\delta \rightarrow 0^{+}} \liminf _{n \rightarrow \infty}\left\{\int_{A_{\delta}^{-}} f\left(v_{1}, z_{n}\right) d x+\int_{A_{\delta}^{+}} f\left(v_{1}, w_{n}\right) d x+\int_{(a, b) \backslash\left(A_{\delta}^{-} \cup A_{\delta}^{+}\right)} f\left(v_{1}^{n}, v_{2}^{n, \delta}\right) d x\right\} \\
& \leq \liminf _{\delta \rightarrow 0^{+}} \liminf _{n \rightarrow \infty}\left\{\int_{A_{\delta}^{-}}\left[\psi\left(v_{1}\right)+\left(z_{n}\right)^{2}\right] d x+\int_{A_{\delta}^{+}}\left[\psi\left(v_{1}\right)+\left(w_{n}\right)^{2}\right] d x+C\left|(a, b) \backslash\left(A_{\delta}^{-} \cup A_{\delta}^{+}\right)\right|\right\} \\
& =\liminf _{\delta \rightarrow 0^{+}}\left\{\int_{A_{\delta}^{-} \cup A_{\delta}^{+}}\left[\psi\left(v_{1}\right)+\left(v_{2}\right)^{2}\right] d x+C\left|(a, b) \backslash\left(A_{\delta}^{-} \cup A_{\delta}^{+}\right)\right|\right\} \\
& =\int_{a}^{b} \psi\left(v_{1}\right) d x+\left(v_{2}\right)^{2}(b-a),
\end{aligned}
$$

where we have used the fact that

$$
\begin{aligned}
\left|(a, b) \backslash\left(A_{\delta}^{-} \cup A_{\delta}^{+}\right)\right| & =\left|\left\{x \in(a, b):\left|\left(v_{1}-1\right)^{2}-\left(v_{1}+1\right)^{2}\right|<\delta\right\}\right|=\left|\left\{x \in(a, b):\left|v_{1}(x)\right|<\delta / 2\right\}\right| \\
& \rightarrow\left|\left\{x \in(a, b): v_{1}(x)=0\right\}\right|=0
\end{aligned}
$$

as $\delta \rightarrow 0^{+}$.

The research of A. Braides was partially supported by the CNR research project "Equazioni Differenziali e Calcolo delle Variazioni", that of I. Fonseca by the National Science Foundation under Grant No. DMS-9731957 and through the Center for Nonlinear Analysis under Grant No. DMS-9803791, and that of G. Leoni by GNAFA, by MURST, Project "Metodi Variazionali ed Equazioni Differenziali Non Lineari", by the Italian CNR, through the strategic project "Metodi e modelli per la Matematica e l'Ingegneria". G. Leoni would like to thank the hospitality and support of the Center for Nonlinear Analysis and of S.I.S.S.A, where part of this work was carried out.

\section{REFERENCES}

[1] E. Acerbi and N. Fusco, Semicontinuity problems in the calculus of variations. Arch. Rational Mech. Anal. 86 (1984) $125-145$.

[2] M. Amar and V. De Cicco, Relaxation of quasi-convex integrals of arbitrary order. Proc. Roy. Soc. Edinburgh Sect. A 124 (1994) 927-946.

[3] L. Ambrosio, S. Mortola and V.M. Tortorelli, Functionals with linear growth defined on vector valued $B V$ functions. J. Math. Pures Appl. 70 (1991) 269-323.

[4] E.J. Balder, A general approach to lower semicontinuity and lower closure in optimal control theory. SIAM J. Control Optim. 22 (1984) 570-598.

[5] J.M. Ball, A version of the fundamental theorem for Young measures, in PDE's and Continuum Models of Phase Transitions, edited by M. Rascle, D. Serre and M. Slemrod. Springer-Verlag, Berlin, Lecture Notes in Phys. 344 (1989) 207-215.

[6] J.M. Ball and F. Murat, Remarks on Chacon's biting lemma. Proc. Amer. Math. Soc. 107 (1989) 655-663.

[7] H. Berliocchi and J.M. Lasry, Intégrands normales et mesures paramétrées en calcul des variations. Bull. Soc. Math. France 101 (1973) 129-184.

[8] A. Braides, A homogenization theorem for weakly almost periodic functionals, Rend. Accad. Naz. Sci. XL Mem. Sci. Fis. Natur. (5) 104 (1986) 261-281.

[9] A. Braides, Relaxation of functionals with constraints on the divergence. Ann. Univ. Ferrara Ser. VII (N.S.) 33 (1987) $157-177$.

[10] A. Braides and A. Defranceschi, Homogenization of Multiple Integrals. Clarendon Press, Oxford (1998).

[11] A. Braides, A. Defranceschi and E. Vitali, Homogenization of free-discontinuity problems. Arch. Rational Mech. Anal. 135 (1996) 297-356.

[12] G. Buttazzo, Semicontinuity, relaxation and integral representation problems in the Calculus of Variations. Longman, Harlow, Pitman Res. Notes Math. Ser. 207 (1989).

[13] B. Dacorogna, Direct Methods in the Calculus of Variations. Springer-Verlag, Berlin (1989).

[14] B. Dacorogna, Weak Continuity and Weak Lower Semicontinuity for Nonlinear Functionals. Springer-Verlag, Berlin, Lecture Notes in Math. 922 (1982). 
[15] G. Dal Maso, An Introduction to $\Gamma$-Convergence. Birkhäuser, Boston (1993).

[16] G. Dal Maso, A. Defranceschi and E. Vitali (private communication).

[17] A. De Simone, Energy minimizers for large ferromagnetic bodies. Arch. Rational Mech. Anal. 125 (1993) 99-143.

[18] I. Fonseca, The lower quasiconvex envelope of the stored energy function for an elastic crystal. J. Math. Pures Appl. 67 (1988) 175-195.

[19] I. Fonseca, G. Leoni, J. Malý and R. Paroni (in preparation).

[20] I. Fonseca and S. Müller, Quasiconvex integrands and lower semicontinuity in L1. SIAM J. Math. Anal. 23 (1992) $1081-1098$.

[21] I. Fonseca and S. Müller, Relaxation of quasiconvex functionals in $B V\left(\Omega, \mathbb{R}^{p}\right)$ for integrands $f(x, u, \nabla u)$. Arch. Rational Mech. Anal. 123 (1993) 1-49.

[22] I. Fonseca and S. Müller, A-quasiconvexity, lower semicontinuity and Young measures. SIAM J. Math. Anal. 30 (1999) 13551390.

[23] N. Fusco, Quasi-convessitá e semicontinuitá per integrali di ordine superiore. Ricerche Mat. 29 (1980) $307-323$.

[24] M. Giaquinta and G. Modica, Regularity results for some classes of higher order non linear elliptic systems. J. reine angew. Math. 311/312 (1979) 145-169.

[25] M. Guidorzi and L. Poggiolini, Lower semicontinuity for quasiconvex integrals of higher order. NoDEA Nonlinear Differential Equations Appl. 6 (1999) 227-246.

[26] J. Kristensen, Finite functionals and Young measures generated by gradients of Sobolev functions. Mathematical Institute, Technical University of Denmark, Mat-Report No. 1994-34 (1994).

[27] P. Marcellini, Approximation of quasiconvex functions and semicontinuity of multiple integrals. Manuscripta Math. 51 (1985) $1-28$.

[28] P. Marcellini and C. Sbordone, Semicontinuity problems in the Calculus of Variations. Nonlinear Anal. 4 (1980) $241-257$.

[29] N.G. Meyers, Quasi-convexity and lower semi-continuity of multiple variational integrals of any order. Trans. Amer. Math. Soc. 119 (1965) 125-149.

[30] C.B. Morrey, Multiple Integrals in the calculus of Variations. Springer-Verlag, Berlin (1966).

[31] S. Müller, Variational models for microstructures and phase transitions, in Calculus of Variations and Geometric Evolution Problems, edited by S. Hildebrant et al. Springer-Verlag, Berlin, Lecture Notes in Math. 1713 (1999) 85-210.

[32] F. Murat, Compacité par compensation : condition nécessaire et suffisante de continuité faible sous une hypothèse de rang constant. Ann. Scuola Norm. Sup. Pisa Cl. Sw. (4) 8 (1981) 68-102.

[33] P. Pedregal, Parametrized Measures and Variational Principles. Birkhäuser, Boston (1997).

[34] L. Tartar, Compensated compactness and applications to partial differential equations, in Nonlinear Analysis and Mechanics: Heriot-Watt Symposium, edited by R. Knops. Longman, Harlow, Pitman Res. Notes Math. Ser. 39 (1979) 136-212.

[35] L. Tartar, The compensated compactness method applied to systems of conservation laws, in Systems of Nonlinear Partial Differential Eq., edited by J.M. Ball. Riedel (1983).

[36] L. Tartar, Étude des oscillations dans les équations aux dérivées partielles nonlinéaires. Springer-Verlag, Berlin, Lectures Notes in Phys. 195 (1984) 384-412.

[37] L. Tartar, $H$-measures, a new approach for studying homogenisation, oscillations and concentration effects in partial differential equations. Proc. Roy. Soc. Edinburgh Sect. A 115 (1990) 193-230.

[38] L. Tartar, On mathematical tools for studying partial differential equations of continuum physics: $H$-measures and Young measures, in Developments in Partial Differential Equations and Applications to Mathematical Physics, edited by Buttazzo, Galdi and Zanghirati. Plenum, New York (1991).

[39] L. Tartar, Some remarks on separately convex functions, in Microstructure and Phase Transitions, edited by D. Kinderlehrer, R.D. James, M. Luskin and J.L. Ericksen. Springer-Verlag, IMA J. Math. Appl. 54 (1993) 191-204.

[40] L.C. Young, Lectures on Calculus of Variations and Optimal Control Theory. W.B. Saunders (1969). 